\title{
Vision-based structural displacement measurement: system performance evaluation and influence factor analysis
}

\author{
X. W. Ye*, Ting-Hua Yi, C. Z. Dong, T. Liu \\ 1st author: \\ Dr. X. W. Ye (Corresponding author) \\ Associate Professor \\ Department of Civil Engineering \\ Zhejiang University \\ Hangzhou 310058, China \\ Tel.: +86-571-88208478 \\ Fax: +86-571-88208685 \\ E-mail: cexwye@zju.edu.cn \\ 2nd author: \\ Prof. Ting-Hua Yi \\ Professor \\ School of Civil Engineering \\ Dalian University of Technology \\ Dalian 116023, China
}

3rd author:

Mr. C. Z. Dong

MSc Candidate

Department of Civil engineering

Zhejiang University

Hangzhou 310058, China

4th author:

Mr. T. Liu

MSc Candidate

Department of Civil engineering

Zhejiang University

Hangzhou 310058, China 


\title{
Vision-based structural displacement measurement: system performance evaluation and influence factor analysis
}

\author{
X. W. Ye ${ }^{\mathrm{a}, *}$, Ting-Hua Yi ${ }^{\mathrm{b}}$, C. Z. Dong ${ }^{\mathrm{a}}$, T. Liu ${ }^{\mathrm{a}}$ \\ ${ }^{a}$ Department of Civil Engineering, Zhejiang University, Hangzhou 310058, China \\ ${ }^{\mathrm{b}}$ School of Civil Engineering, Dalian University of Technology, Dalian 116023, China \\ * Corresponding author. E-mail address: cexwye@zju.edu.cn (X. W. Ye).
}

\begin{abstract}
In the past decade, the emerging machine vision-based measurement technology has gained great concerns among civil engineers due to its overwhelming merits of non-contact, long-distance, and high-resolution. A critical issue regarding to the measurement performance and accuracy of the vision-based system is how to identify and eliminate the systematic and unsystematic error sources. In this paper, a vision-based structural displacement measurement system integrated with a digital image processing approach is developed. The performance of the developed vision-based system is evaluated by comparing the results simultaneously obtained by the vision-based system and those measured by the magnetostrictive displacement sensor (MDS). A series of experiments are conducted on a shaking table to examine the influence factors which will affect the accuracy and stability of the vision-based system. It is demonstrated that illumination and vapor have a critical effect on the measurement results of the vision-based system.
\end{abstract}

Keywords: structural health monitoring; vision-based system; digital image processing; multi-point pattern matching algorithm; influence factor analysis 


\section{Introduction}

In recent years, the machine vision technology has been widely employed in measurement of structural dynamic behaviors and received increasing concerns from the community of structural health monitoring (SHM) [1-7]. With the aid of the digital image processing technique and modern computer and information science, a significant number of vision-based systems have been developed and applied to measure the structural dynamic displacement [8]. Lee et al. [9] used the digital image processing technique to monitor the real-time displacement of a flexible bridge. Fukuda et al. [10] developed a time synchronization vision-based system for measurement of multi-point structural displacements. Choi et al. [11] introduced a vision-based displacement measurement system and the accuracy of measurement was improved by use of the region of interest (ROI) in the digital image. Santos et al. [12] investigated the calibration problem of vision-based system in displacement monitoring of suspension bridges. Jeon et al. [13] developed a vision-based remote 6-DOF structural displacement monitoring system with high accuracy in real time measurement. Park et al. [14] proposed a 3D displacement measurement model for health monitoring of structures using a motion capture system with multiple cameras.

To achieve the accurate and reliable measurement results by the vision-based system, it is a necessity to carefully examine the error sources and further to make effective measures. Recently, research efforts have been devoted to investigating the factors influencing the performance of the vision-based system, such as subset size [15-17], gray level interpolation [18], and correlation functions or shape functions $[19,20]$. Yoneyama and Kikuta [21] proposed a method of lens distortion correction in order to improve the measurement accuracy of digital image correlation for two-dimensional displacement measurement. Ma et al. [22] investigated the systematic 
strain measurement error in digital image correlation caused by self-heating of digital cameras. Fazzini et al. [23] studied the image characteristics on digital image correlation error assessment. Zhou et al. [24] presented a method of adaptive subset offset for systematic error reduction in incremental digital image correlation.

This paper presents a non-contact vision-based structural displacement measurement system which can realize quasi-distributed displacement measurement of structures. A series of experiments are carried out on a shaking table to evaluate the system performance through the comparative study between the results obtained by the developed vision-based system and those simultaneously measured by the magnetostrictive displacement sensor (MDS). The effects of the influencing factors on the measurement accuracy of the developed vision-based system are investigated including the environmental illumination, elevation angle of the digital camera, and vapor surrounding the targets.

\section{Vision-based method for multi-point structural displacement measurement}

\subsection{Multi-point pattern matching algorithm}

The vision-based multi-point structural displacement measurement is realized based on multi-point pattern matching algorithm. In the first grayscale image, the patterns are predefined where the measurement targets are incorporated. The action of pattern matching is a process of searching the measurement targets in the subsequent images captured by the digital camera. In this process, the pre-designated patterns with the measurement targets will wander on the subsequent images. The scores representing the matching extent between the patterns and the corresponding regions of the captured images will be calculated on the basis of multi-point pattern matching algorithm. When the scores of the matching tasks reach the maximum values, it indicates that the patterns 
best correspond with the regions of the captured images and meanwhile the target positions are identified [25].

As illustrated in Fig. 1, the sub-image is extracted to represent the pattern $f_{k}(x, y)$ with the size of $m_{k} \times n_{k}$ in the initial image $g^{0}(x, y)$ with the size of $M \times N$. Here, $k$ is the number of the patterns. The initial coordinate of the center point of the $k$ th pattern is denoted as $\left(x_{k}^{0}, y_{k}^{0}\right)$. The correlation coefficient $c_{k}(i, j)$ between the pattern $f_{k}(x, y)$ and the image $g^{t}(x, y)$ at the point $\left(i_{k}, j_{k}\right)$ is given by

$$
c_{k}(i, j)=\sum_{x=0}^{M-1} \sum_{y=0}^{N-1} f_{k}(x, y) g^{t}(x+i, y+j)
$$

where $i=0,1, \ldots, M-1, j=0,1, \ldots, N-1$, and $t$ is the time on which the image is captured. Assuming that the origin of the image $g^{t}(x, y)$ is at the top left corner, the correlation computation is the process of moving the pattern $f_{k}(x, y)$ on the image and computing the correlation coefficient $c_{k}(i, j)$. The grayscale value of each pixel in the pattern is multiplied by that in the overlapped region of the image, and the summation is executed for the overlapped region between $f_{k}(x, y)$ and $g^{t}(x, y)$. The maximum value of $c_{k}(i, j)$ indicates that $f_{k}(x, y)$ best matches the overlapped region of $g^{t}(x, y)$ and the target position is identified. The coordinate of the center point of this overlapped region is represented by $\left(x_{k}^{t}, y_{k}^{t}\right)$.

\section{Fig. 1. Correlation between patterns and captured image}

The above-described correlation coefficient is very sensitive to the amplitude variation of geometrical size and grayscale value of the pattern and image. This sensitivity problem can be resolved by computing the normalized correlation coefficient $\alpha_{k}(i, j)$, which is defined as [26] 


$$
\alpha_{k}(i, j)=\frac{\sum_{x=0}^{M-1} \sum_{y=0}^{N-1}\left(f_{k}(x, y)-\overline{f_{k}}\right)\left(g^{t}(x+i, y+j)-\overline{g^{t}}\right)}{\left[\sum_{x=0}^{M-1} \sum_{y=0}^{N-1}\left(f_{k}(x, y)-\overline{f_{k}}\right)\right]^{\frac{1}{2}}\left[\sum_{x=0}^{M-1} \sum_{y=0}^{N-1}\left(g^{t}(x+i, y+j)-\overline{g^{t}}\right)\right]^{\frac{1}{2}}}
$$

where $\bar{f}_{k}$ is the average grayscale value of the pixels in the template $f_{k}(x, y)$, and $\bar{g}_{k}$ is the average grayscale value of the overlapped region between $f_{k}(x, y)$ and $g^{t}(x, y)$. The value of $\alpha_{k}(i, j)$ is ranged from -1 to 1 , which is independent of the scale change of $f_{k}(x$, $y$ ) and $g^{t}(x, y)$. The maximum value of the normalized correlation coefficient $\alpha_{k}(i, j)$ represents the best pattern matching is realized.

Generally, the target moves within a certain region of the image named as region of interest $(\mathrm{ROI}), w^{t}(x, y)$, and therefore it is unnecessary to perform the pattern matching task in the whole image. To improve the matching efficiency, the pattern matching can be conducted within the ROI, and the normalized correlation coefficient $\beta_{k}(i, j)$ can be represented by

$$
\beta_{k}(i, j)=\frac{\sum_{x=0}^{M-1} \sum_{y=0}^{N-1}\left(f_{k}(x, y)-\overline{f_{k}}\right)\left(w^{t}(x+i, y+j)-\overline{w^{t}}\right)}{\left[\sum_{x=0}^{M-1} \sum_{y=0}^{N-1}\left(f_{k}(x, y)-\overline{f_{k}}\right)\right]^{\frac{1}{2}}\left[\sum_{x=0}^{M-1} \sum_{y=0}^{N-1}\left(w^{t}(x+i, y+j)-\overline{w^{t}}\right)\right]^{\frac{1}{2}}}
$$

where $\bar{w}_{k}$ is the average grayscale value of the pixels in the ROI.

\subsection{Scale transformation and displacement calculation}

When the best pattern matching status is accomplished in the image coordinate, it is required to convert the change of the target position in the image to the actual structural displacement. If there are $h$ pixels in the vertical direction for the target in the image and the actual size of the target in the vertical direction is $H$, and then the scale ratio $r$ can be calculated by 


$$
r=\frac{H}{h}
$$

After obtaining the initial coordinate of the center point of the $k$ th pattern $\left(x^{0}{ }_{k}, y^{0}{ }_{k}\right)$ and the coordinate of the center point of the overlapped region $\left(x_{k}^{t}, y_{k}^{t}\right)$, the horizontal structural displacement $x_{k}(t)$ and the vertical structural displacement $y_{k}(t)$ of the $k$ th target can be derived by

$$
\left[\begin{array}{l}
x_{k}(t) \\
y_{k}(t)
\end{array}\right]=r\left[\begin{array}{cc}
x_{k}^{t} & x_{k}^{0} \\
y_{k}^{t} & y_{k}^{0}
\end{array}\right]\left[\begin{array}{cc}
1 & 1 \\
-1 & -1
\end{array}\right]
$$

\section{Experimental verification and analysis of influence factors}

\subsection{System configuration}

As illustrated in Fig. 2, the vision-based multi-point structural displacement measurement system consists of a high-resolution industrial charge-coupled device (CCD) camera, a zoom lens, a computer, and a Gigabit Ethernet standard LAN wire. In this system, a Prosilica GE1050 camera with a Navidar 12X zoom extender lens serves as the image acquisition equipment.

Fig. 2. Vision-based system for multi-point structural displacement measurement

\subsection{Experimental evaluation of system performance: effect of illumination}

Illumination is one of the most important environmental factors which will affect the quality of captured images and the sampling efficiency of the vision-based system. Basically, an environment with adequate illumination is desired to acquire satisfactory results of structural displacement. Under an inferior light condition, it is not easy for the digital camera to capture distinct images even though much time of exposure is taken. In this connection, the task of pattern matching will not be effectively fulfilled. In real-world applications, the vision-based system is liable to confront different kinds of illumination conditions which may make the measurement results unstable and 
inaccurate.

As shown in Table 1, in this study, a total of five levels of illumination (600 lux, $450 \operatorname{lux}, 300 \operatorname{lux}, 150$ lux and 75 lux) are configured to investigate the effect of illumination on the measurement accuracy of the vision-based system. As illustrated in Fig. 3, the comparative experiments are conducted on a self-made shaking table on which a rigid board attached with quick response $(\mathrm{QR})$ codes is fixed. Two QR codes ( $\mathrm{P} 1$ and $\mathrm{P} 2$ ) are regarded as the measurement targets for the vision-based system, as shown in Fig. 4. An MDS is also installed to measure the displacement of the two targets. An illumination sensor is deployed nearby the targets to record the level of illumination. The illumination condition is adjusted by switching the lamps in the room. The displacements of the targets during the vibration process of the shaking table are simultaneously measured by the vision-based system and the MDS. The vibration frequency of the shaking table is $0.3 \mathrm{~Hz}$ and the sampling frequency of the two systems is $10 \mathrm{~Hz}$. The distance between the digital camera and the targets is $2 \mathrm{~m}$.

Table 1. Test cases for study of illumination effect

Fig. 3. Experimental setup for study of illumination effect

\section{Fig. 4. Targets board with QR codes}

Figs. 5 show the images captured by the CCD camera in the aforementioned five cases. The exposure value and the gain value of the CCD camera are 90,000 and 22, respectively. In general, the gray level and quality of the image will be changed under different light conditions. When the illumination decreases, the feature of the QR code target tends to be dark which makes it hard to be recognized. In this study, the target is grayish if the level of the illumination is 600 lux and the region around the target is 
bright which makes the target stands out from the surrounding environment. In this circumstance, the target is recognizable and it is an ideal state for pattern matching and structural displacement measurement. When the level of the illumination is 75 lux, the target and the surrounding region are almost integrated so that it is very hard to recognize the target from the surrounding environment.

Fig. 5. Images captured by CCD camera in five cases: (a) Case 1.1, (b) Case 1.2, (c)

\section{Case $1.3,(\mathrm{~d})$ Case 1.4, (e) Case 1.5}

Figs. 6-10 illustrate the displacement time histories of P1 and P2 measured by the vision-based system and the MDS in the aforementioned five cases. It is seen from Figs. 6-10 that in the first four cases the displacement results measured by the vision-based system are in consistent with those obtained by the MDS. However, in the case 1.5, when the level of the illumination is 75 lux, the vision-based system has the difficulty in accomplishing the task of pattern matching and a considerable number of spurious tones are generated. As a result, the inaccurate displacement results are obtained by the vision-based system due to the illumination effect.

Fig. 6. Displacement time histories in case 1.1: (a) P1, (b) P2

Fig. 7. Displacement time histories in case 1.2: (a) P1, (b) P2

Fig. 8. Displacement time histories in case 1.3: (a) P1, (b) P2

Fig. 9. Displacement time histories in case 1.4: (a) P1, (b) P2

Fig. 10. Displacement time histories in case 1.5: (a) P1, (b) P2

\subsection{Experimental evaluation of system performance: effect of elevation angle}

In practical applications, the longitudinal axis of the digital camera and lens may 
not be perpendicular to the object and an elevation angle will be existed. In vision-based structural displacement measurement, the pixel calibration and pattern matching may be affected by the perspective error. In this study, the effect of the elevation angle on the measurement accuracy of the vision-based system is carried out. A total of three kinds of elevation angles $\left(5^{\circ}, 10^{\circ}\right.$ and $\left.15^{\circ}\right)$ of the digital camera and lens are employed, as illustrated in Table 2.

\section{Table 2. Test cases for study of elevation angle effect}

Fig. 11 shows the experimental setup for study of the elevation angle effect. Figs. 12-14 illustrate the displacement time histories of $\mathrm{P} 1$ and $\mathrm{P} 2$ measured by the vision-based system and the MDS in the aforementioned three cases. The displacement time histories obtained from the two systems agree well with each other. However, in the real situation of field measurement, a larger elevation angle of the digital camera and lens is probably existed and may affect the measurement accuracy of the vision-based system.

Fig. 11. Experimental setup for study of elevation angle effect

Fig. 12. Displacement time histories in case 2.1: (a) P1, (b) P2

Fig. 13. Displacement time histories in case 2.2: (a) P1, (b) P2

Fig. 14. Displacement time histories in case 2.3: (a) P1, (b) P2

\subsection{Experimental evaluation of system performance: effect of vapor}

The system performance of the vision-based system also will be affected by the bad weather (e.g., fog) in practical applications. Fog is able to shelter the target so that the digital camera has the difficulty in tracing the target during the displacement measurement. In this study, a humidifier is used to generate different levels of vapor to 
simulate the foggy weather, and the effect of the vapor on the measurement accuracy of the vision-based system is investigated. As shown in Table 3, a total of four cases are arranged by controlling the humidifier, and the temperatures of the humidifier and laboratory room are also recorded. Fig. 15 illustrates the experimental setup for study of the vapor effect.

Table 3. Test cases for study of vapor effect

Fig. 15. Experimental setup for study of vapor effect

Figs. 16-19 illustrate the displacement time histories of P1 and P2 measured by the vision-based system and the MDS in the aforementioned four cases. For the cases 3.1 and 3.2, the displacement time histories obtained by the vision-based system agree well with those obtained from the MDS. For the case 3.3, the results of P1 are satisfactory; while the displacement time history of P2 measured by the vision-based system is not acceptable. For the case 3.4, the results of P1 and P2 measured by the vision-based system are contaminated by a large number of burrs in comparison with those measured by the MDS. This indicates that the vapor has a great effect on the measurement accuracy of the vision-based system.

Fig. 16. Displacement time histories in case 3.1: (a) P1, (b) P2

Fig. 17. Displacement time histories in case 3.2: (a) P1, (b) P2

Fig. 18. Displacement time histories in case 3.3: (a) P1, (b) P2

Fig. 19. Displacement time histories in case 3.4: (a) P1, (b) P2

\section{Conclusions}

A vision-based system for multi-point structural displacement measurement has 
been developed. Experimental research was carried out on a shaking table, and the performance of the developed vision-based system was verified by a comparative study between the vision-based system and the MDS. Through analyzing the influence factors, it is indicated that the measurement accuracy and stability of the vision-based system will be affected by illumination and vapor. A self-adaptive algorithm is a possible way to solve the illumination-induced adverse effect on the measurement accuracy, and the shelter problem caused by the vapor can be improved by use of a predictive algorithm. In this study, the effect of the elevation angle of the digital camera on the measurement results of the vision-based system is not sensitive, while attentions should be attached during field measurement if a larger elevation angle of the digital camera is existed. In the further research on this issue, the effects of other influence factors (e.g., wind, rain, ground vibration, etc.) on the measurement performance of the vision-based system will be examined.

\section{Acknowledgements}

The work described in this paper was jointly supported by the National Science Foundation of China (Grant Nos. 51308493 and U1234204), the Research Fund for the Doctoral Program of Higher Education of China (Grant No. 20130101120080), the Fok Ying Tong Education Foundation (Grant No. 141072), and the Science Fund for Distinguished Young Scholars of Dalian (Grant No. 2014J11JH125).

\section{References}

[1] A.M. Wahbeh, J.P. Caffrey, S.F. Masri, A vision-based approach for the direct measurement of displacements in vibrating systems, Smart Materials and Structures $12(5)(2003) 785-794$.

[2] Y.Q. Ni, X.W. Ye, J.M. Ko, Monitoring-based fatigue reliability assessment of steel 
bridges: analytical model and application, Journal of Structural Engineering, ASCE 136 (12) (2010) 1563-1573.

[3] Y.Q. Ni, X.W. Ye, J.M. Ko, Modeling of stress spectrum using long-term monitoring data and finite mixture distributions, Journal of Engineering Mechanics, ASCE 138 (2) (2012) 175-183.

[4] X.W. Ye, Y.Q. Ni, K.Y. Wong, J.M. Ko, Statistical analysis of stress spectra for fatigue life assessment of steel bridges with structural health monitoring data, Engineering Structures 45 (2012) 166-176.

[5] H.S. Park, J.Y. Kim, J.G. Kim, S.W. Choi, Y. Kim, A new position measurement system using a motion-capture camera for wind tunnel tests, Sensors 13 (9) (2013) 12329-12344.

[6] C. Cheng, K. Kawaguchi, A preliminary study on the response of steel structures using surveillance camera image with vision-based method during the Great East Japan Earthquake, Measurement 62 (2015) 142-168.

[7] D.L.B.R. Jurjo, C. Magluta, N. Roitman, P.B. Gonçalves, Analysis of the structural behavior of a membrane using digital image processing, Mechanical Systems and Signal Processing 54-55 (2015) 394-404.

[8] X.W. Ye, T.H. Yi, C.Z. Dong, T. Liu, H. Bai, Multi-point displacement monitoring of bridges using a vision-based approach, Wind and Structures 20 (2) (2015) 315-326.

[9] J.J. Lee, M. Shinozuka, Real-time displacement measurement of a flexible bridge using digital image processing techniques, Experimental Mechanics, 46 (1) (2006) $105-114$

[10] Y. Fukuda, M.Q. Feng, M. Shinozuka, Cost-effective vision-based system for monitoring dynamic response of civil engineering structures, Structural Control and 
Health Monitoring 17 (8) (2010) 918-936.

[11] H.S. Choi, J.H. Cheung, S.H. Kim, J.H. Ahn, Structural dynamic displacement vision system using digital image processing, NDT \& E International 44 (7) (2011) 597-608.

[12] C.A. Santos, C.O. Costa, J.P. Batista, Calibration methodology of a vision system for measuring the displacements of long-deck suspension bridges, Structural Control and Health Monitoring 19 (3) (2012) 385-404.

[13] H.M. Jeon, Y.J. Kim, D.H. Lee, H. Myung, Vision-based remote 6-DOF structural displacement monitoring system using a unique maker, Smart Structures and Systems 13 (6) (2014) 927-942.

[14] S.W. Park, H.S. Park, J.H. Kim, H. Adeli, 3D displacement measurement model for health monitoring of structures using a motion capture system, Measurement 59 (2015) 352-362.

[15] D. Lecompte, A. Smits, S. Bossuyt, H. Sol, J. Vantomme, D.V. Hemelrijck, A.M. Habraken, Quality assessment of speckle patterns for digital image correlation, Optics and Lasers in Engineering 44 (11) (2006) 1132-1145.

[16] Y.F. Sun, J.H.L. Pang, Study of optimal subset size in digital image correlation of speckle pattern images, Optics and Lasers in Engineering 45 (9) (2007) 967-974.

[17] G. Crammond, S.W. Boyd, J.M. Dulieu-Barton, Speckle pattern quality assessment for digital image correlation, Optics and Lasers in Engineering 51 (2013) $1368-1378$.

[18] H.M. Schreier, J.R. Braasch, M.A. Sutton, Systematic errors in digital image correlation caused by intensity interpolation, Society of Photo-Optical Instrumentation Engineers 39 (11) (2000) 2915-2921.

[19] H.M. Schreier, M.A. Sutton, Systematic errors in digital image correlation due to 
undermatched subset shape functions, Experimental Mechanics 42 (3) (2002) 303-310.

[20] L.P. Yu, B. Pan, The errors in digital image correlation due to overmatched shape functions, Measurement Science and Technology 26 (4) (2015) 045202.

[21] S. Yoneyama, H. Kikuta, A. Kitagawa, K. Kitamura, Lens distortion correction for digital image correlation by measuring rigid body displacement, Optical Engineering 45 (2) (2006) 023602.

[22] S.P. Ma, J.Z. Pang, Q.W. Ma, The systematic error in digital image correlation induced by self-heating of a digital camera, Measurement Science and Technology 23 (2) (2012) 025403.

[23] M. Fazzini, S. Mistou, O. Dalverny, L. Robert, Study of image characteristics on digital image correlation error assessment, Optics and Lasers in Engineering 48 (2010) 335-339.

[24] Y.H. Zhou, C. Sun, J.B. Chen, Adaptive subset offset for systematic error reduction in incremental digital image correlation, Optics and Lasers in Engineering 55 (2014) $5-11$.

[25] R.C. Gonzalez, R.E. Woods (Eds.), Digital Image Processing, 3rd Edition, Pearson Prentice Hall, Upper Saddle River, NJ, 2008.

[26] X.W. Ye, Y.Q. Ni, T.T. Wai, K.Y. Wong, X.M. Zhang, F. Xu, A vision-based system for dynamic displacement measurement of long-span bridges: algorithm and verification, Smart Structures and Systems 12 (3-4) (2013) 363-379. 


\section{List of Figure Captions}

Fig. 1. Correlation between patterns and captured image

Fig. 2. Vision-based system for multi-point structural displacement measurement

Fig. 3. Experimental setup for study of illumination effect

Fig. 4. Targets board with QR codes

Fig. 5. Images captured by CCD camera in five cases: (a) Case 1.1, (b) Case 1.2, (c)

Case 1.3, (d) Case 1.4, (e) Case 1.5

Fig. 6. Displacement time histories in case 1.1: (a) P1, (b) P2

Fig. 7. Displacement time histories in case 1.2: (a) P1, (b) P2

Fig. 8. Displacement time histories in case 1.3: (a) P1, (b) P2

Fig. 9. Displacement time histories in case 1.4: (a) P1, (b) P2

Fig. 10. Displacement time histories in case 1.5: (a) P1, (b) P2

Fig. 11. Experimental setup for study of elevation angle effect

Fig. 12. Displacement time histories in case 2.1: (a) P1, (b) P2

Fig. 13. Displacement time histories in case 2.2: (a) P1, (b) P2

Fig. 14. Displacement time histories in case 2.3: (a) P1, (b) P2

Fig. 15. Experimental setup for study of vapor effect

Fig. 16. Displacement time histories in case 3.1: (a) P1, (b) P2

Fig. 17. Displacement time histories in case 3.2: (a) P1, (b) P2

Fig. 18. Displacement time histories in case 3.3: (a) P1, (b) P2

Fig. 19. Displacement time histories in case 3.4: (a) P1, (b) P2 


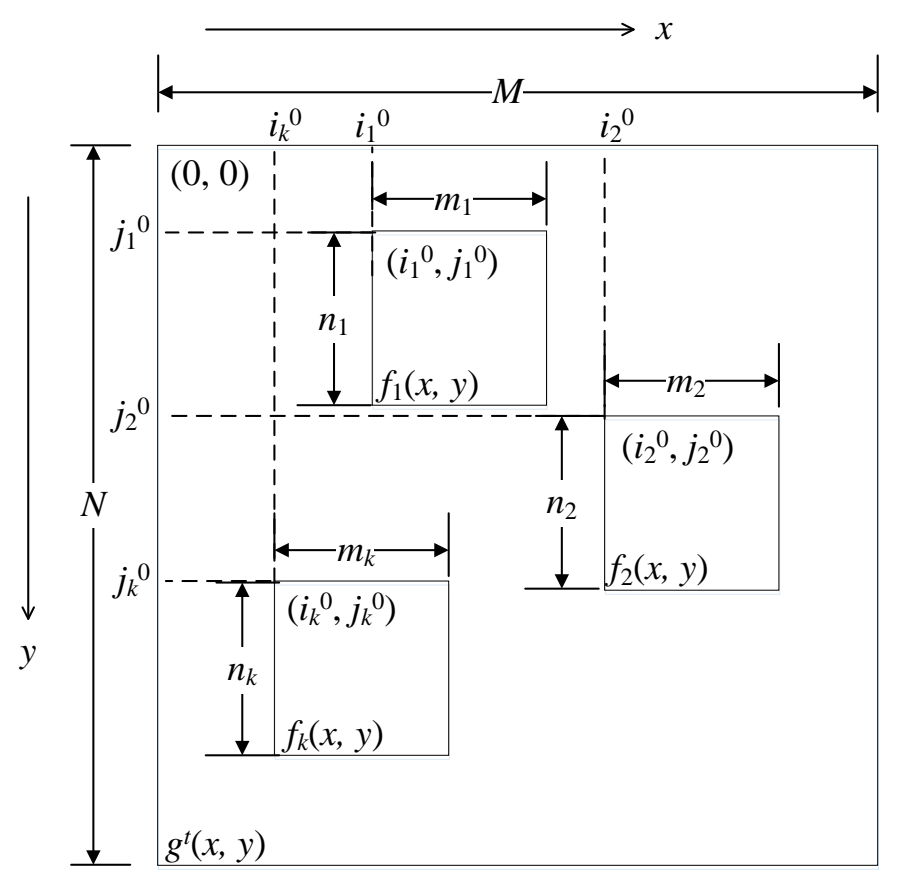

Fig. 1. Correlation between patterns and captured image 


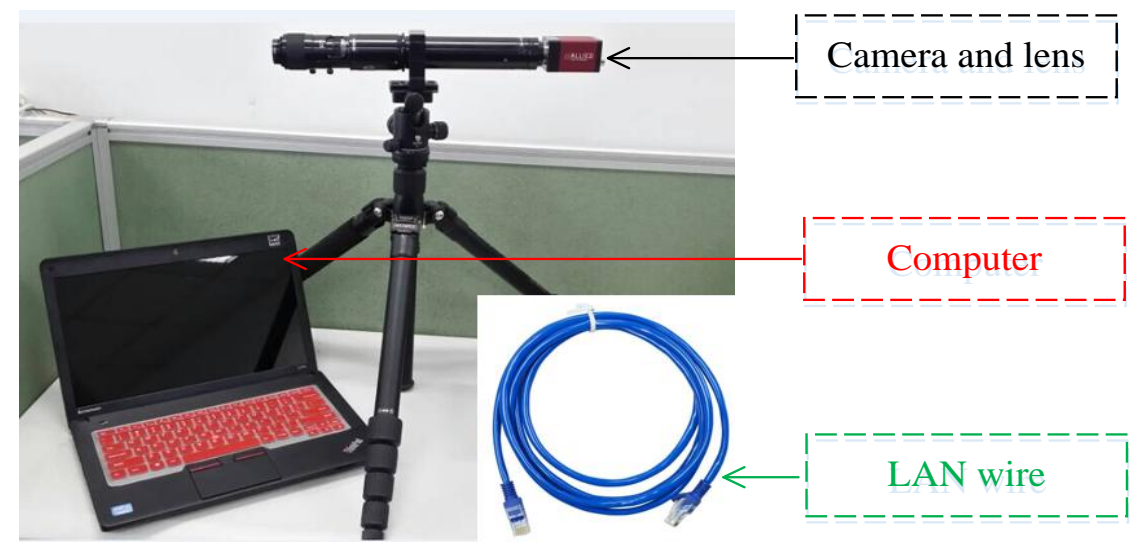

Fig. 2. Vision-based system for multi-point structural displacement measurement 


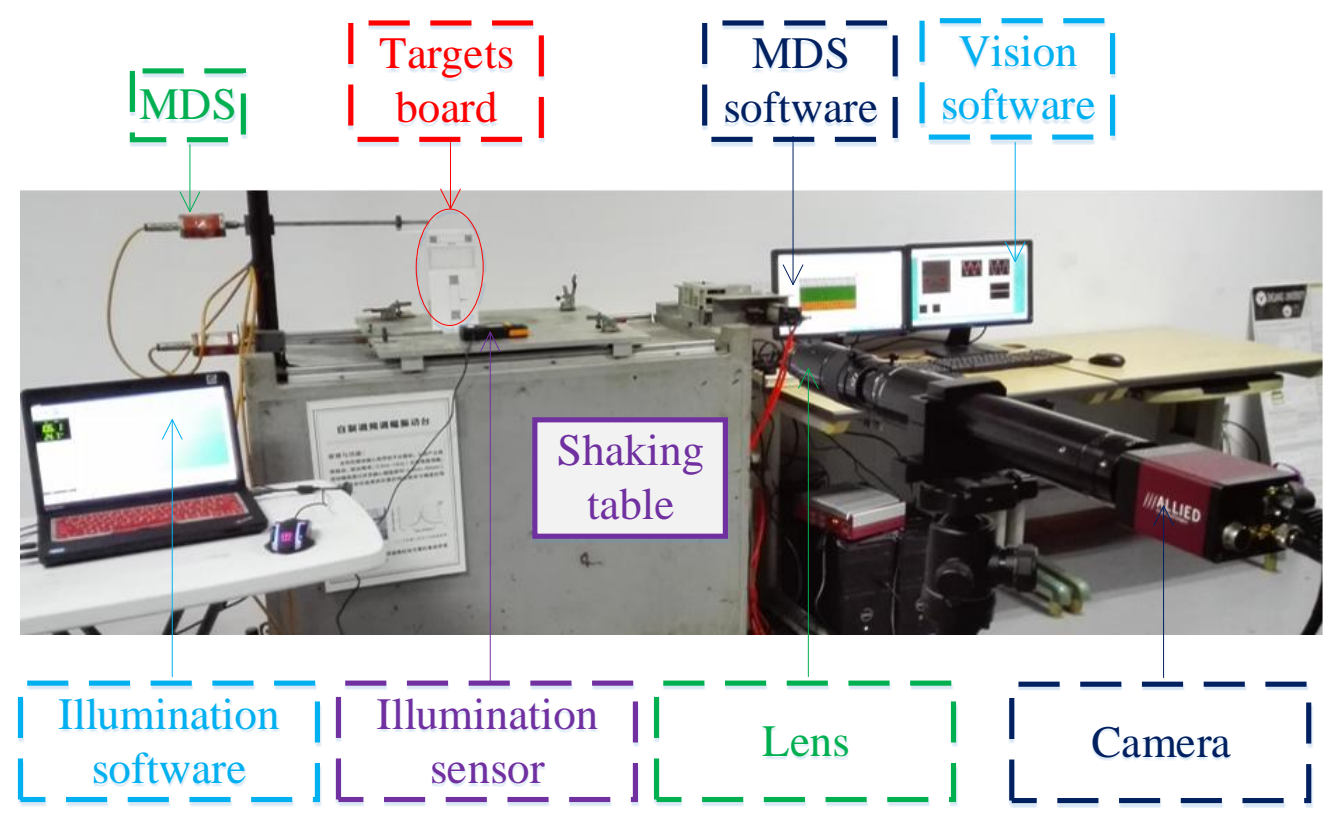

Fig. 3. Experimental setup for study of illumination effect 


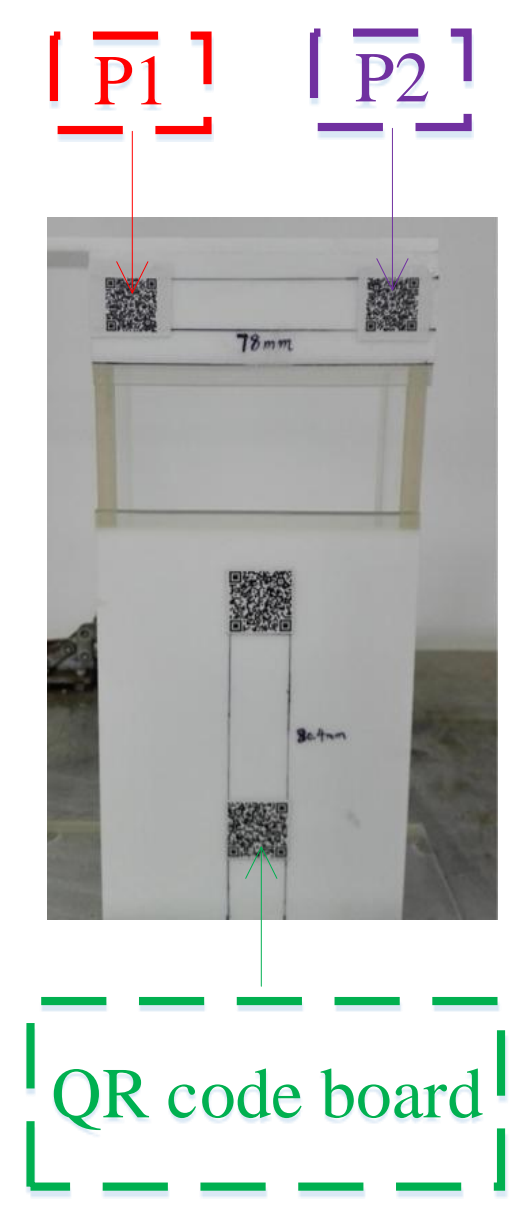

Fig. 4. Targets board with QR codes 


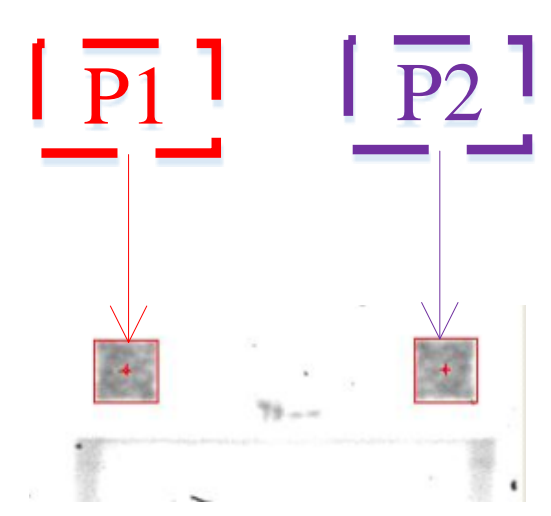

(a)

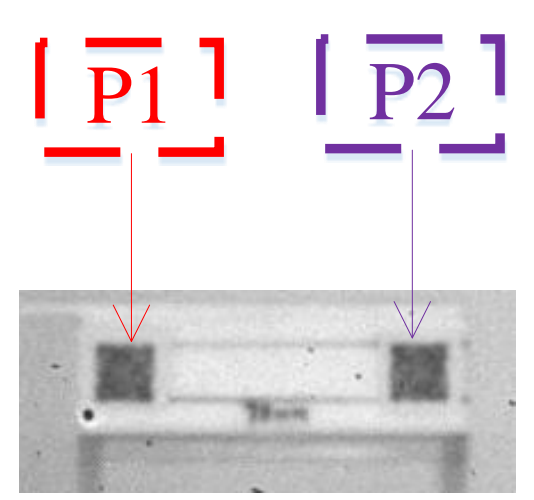

(c)

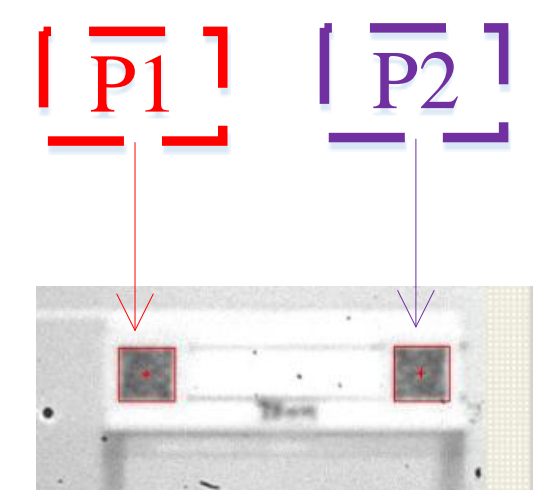

(b)

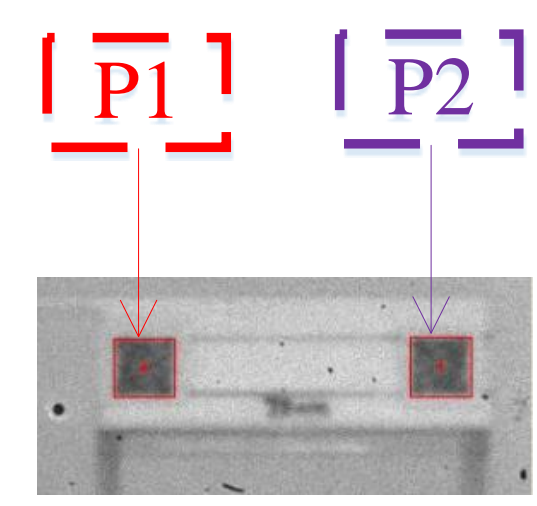

(d)

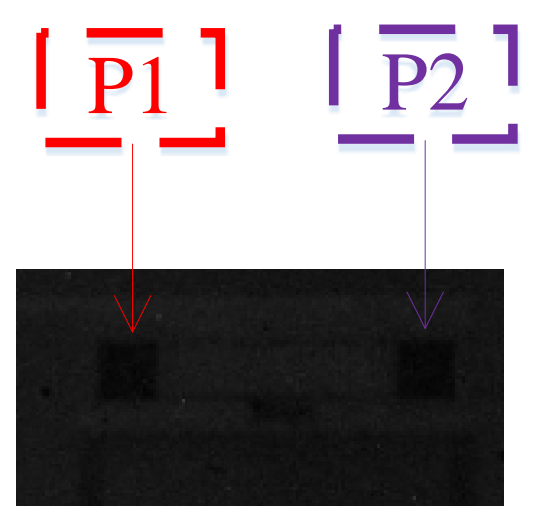

(e)

Fig. 5. Images captured by CCD camera in five cases: (a) Case 1.1, (b) Case 1.2, (c)

Case 1.3, (d) Case 1.4, (e) Case 1.5 


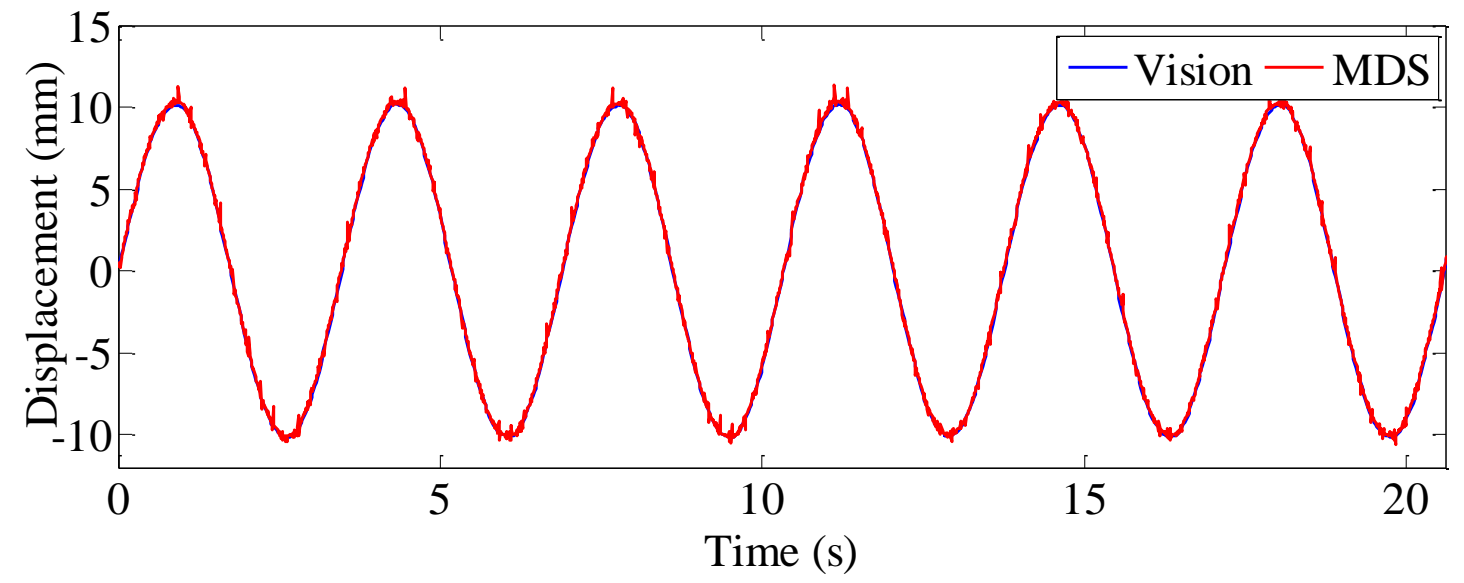

(a)

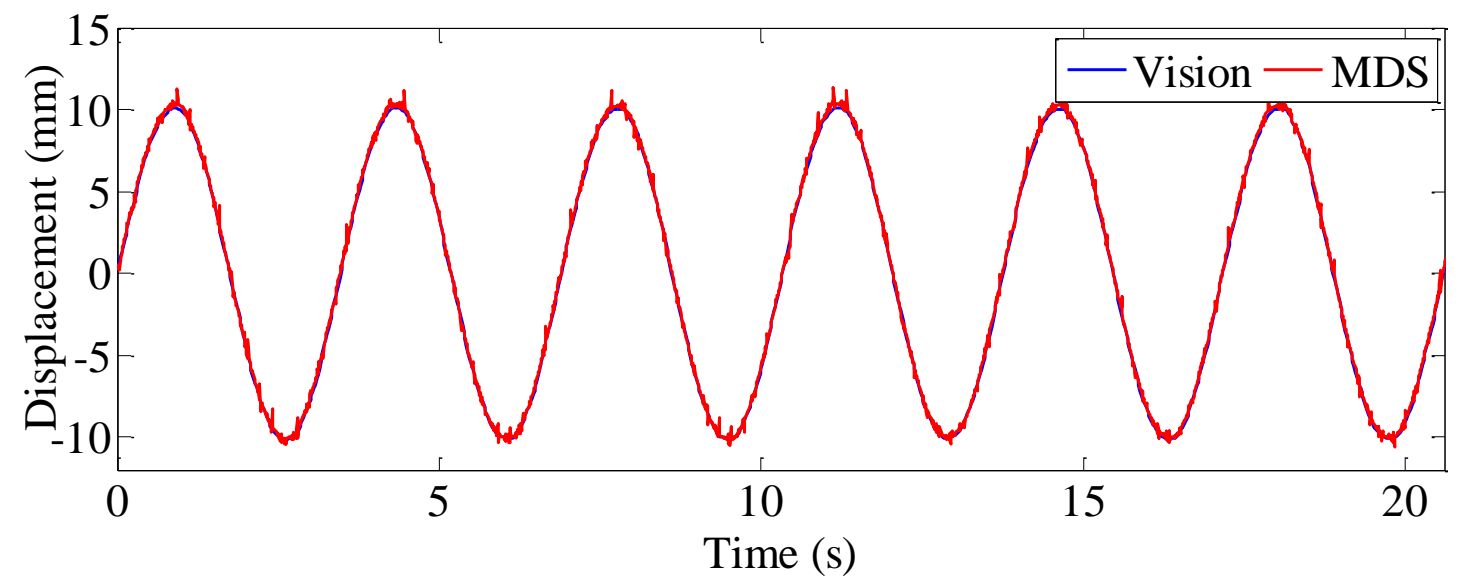

(b)

Fig. 6. Displacement time histories in case 1.1: (a) P1, (b) P2 


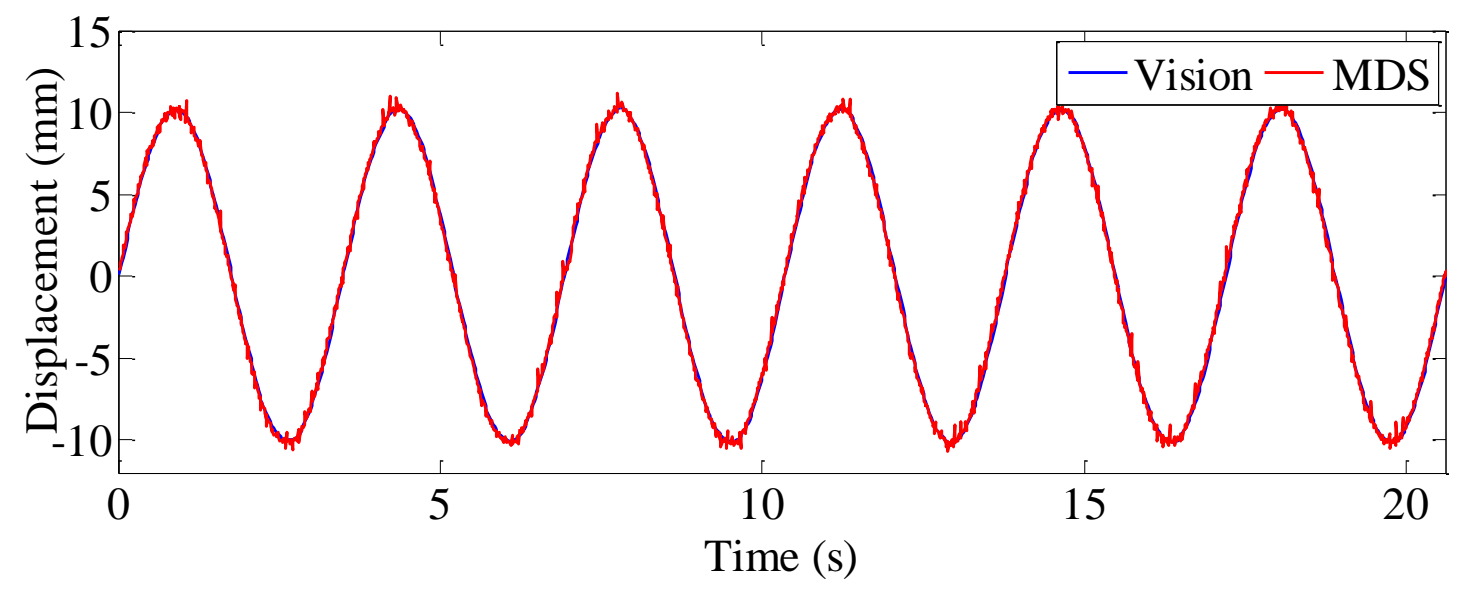

(a)

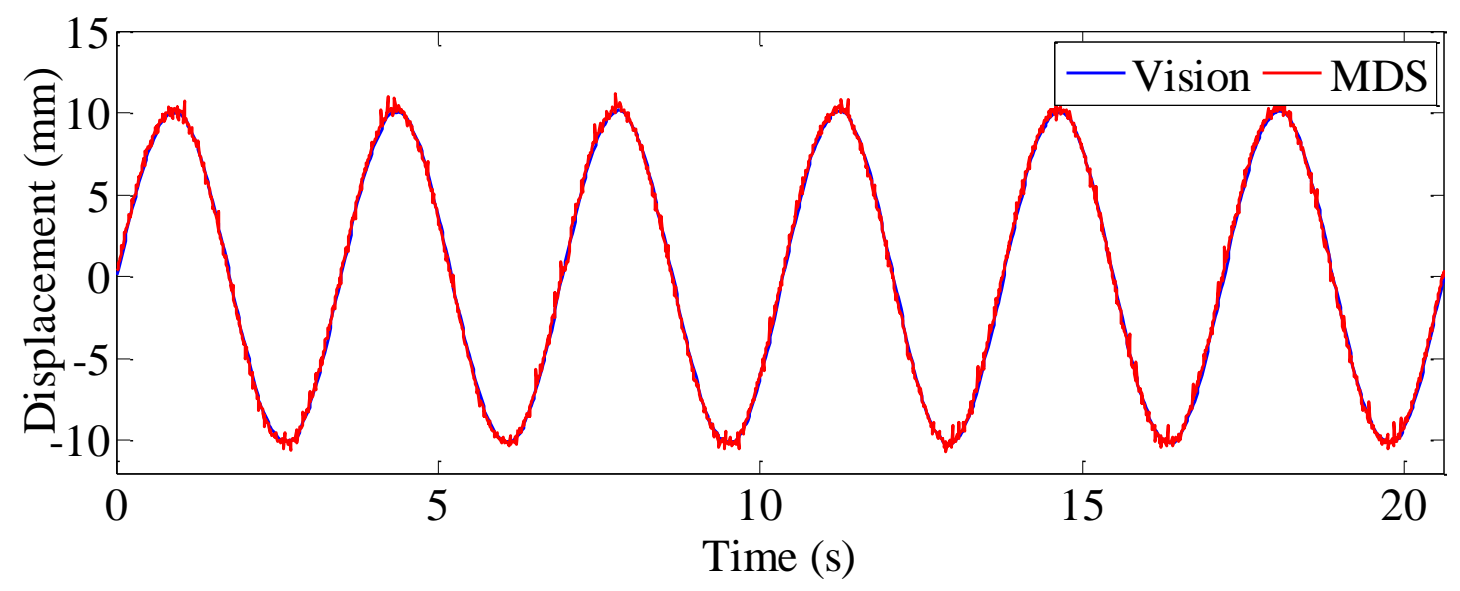

(b)

Fig. 7. Displacement time histories in case 1.2: (a) P1, (b) P2 


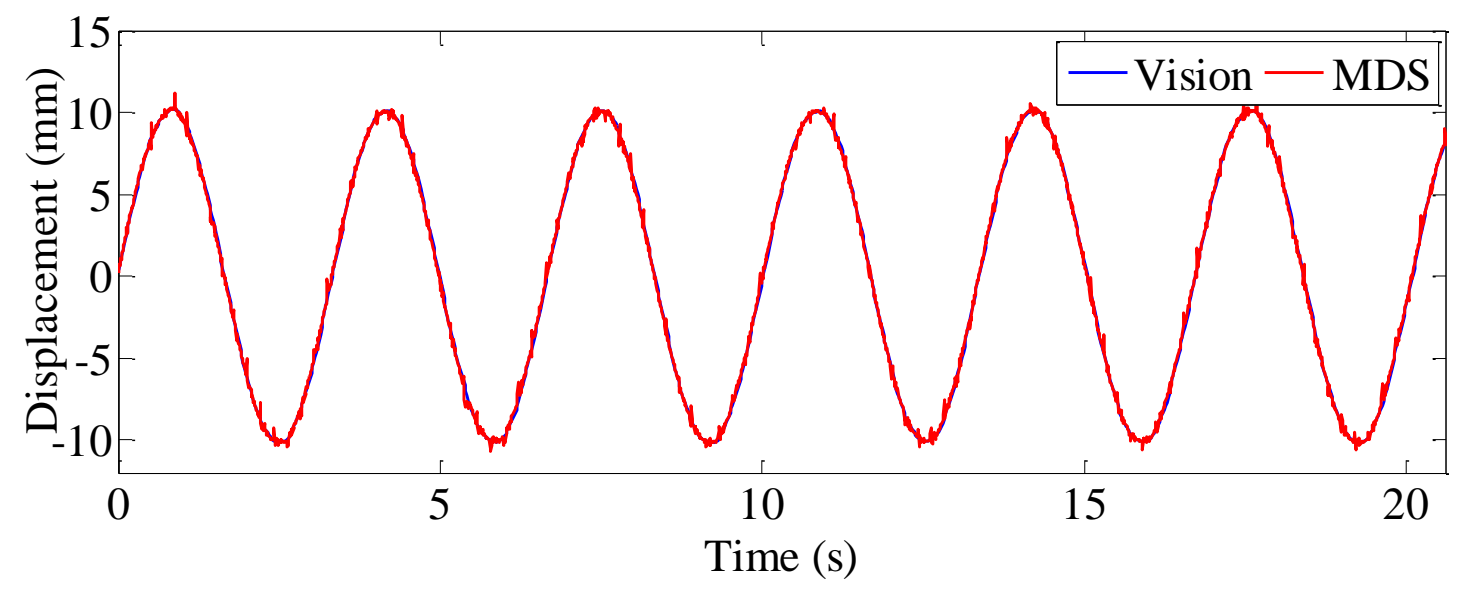

(a)

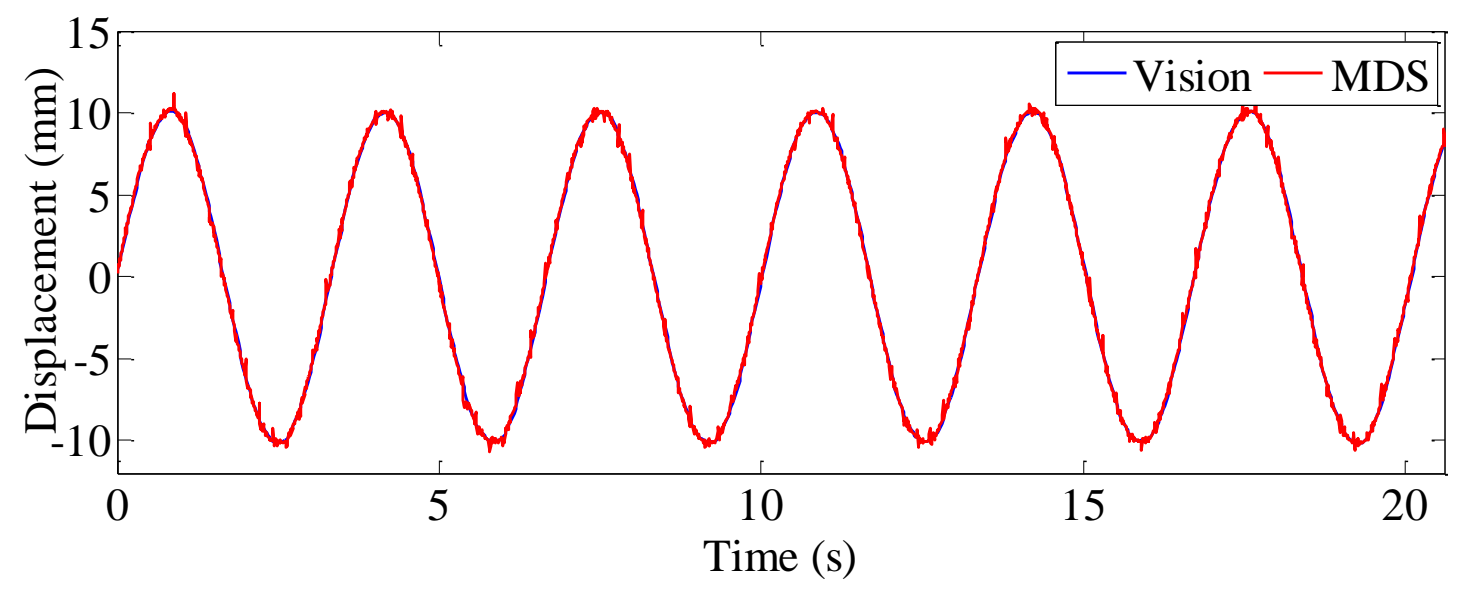

(b)

Fig. 8. Displacement time histories in case 1.3: (a) P1, (b) P2 


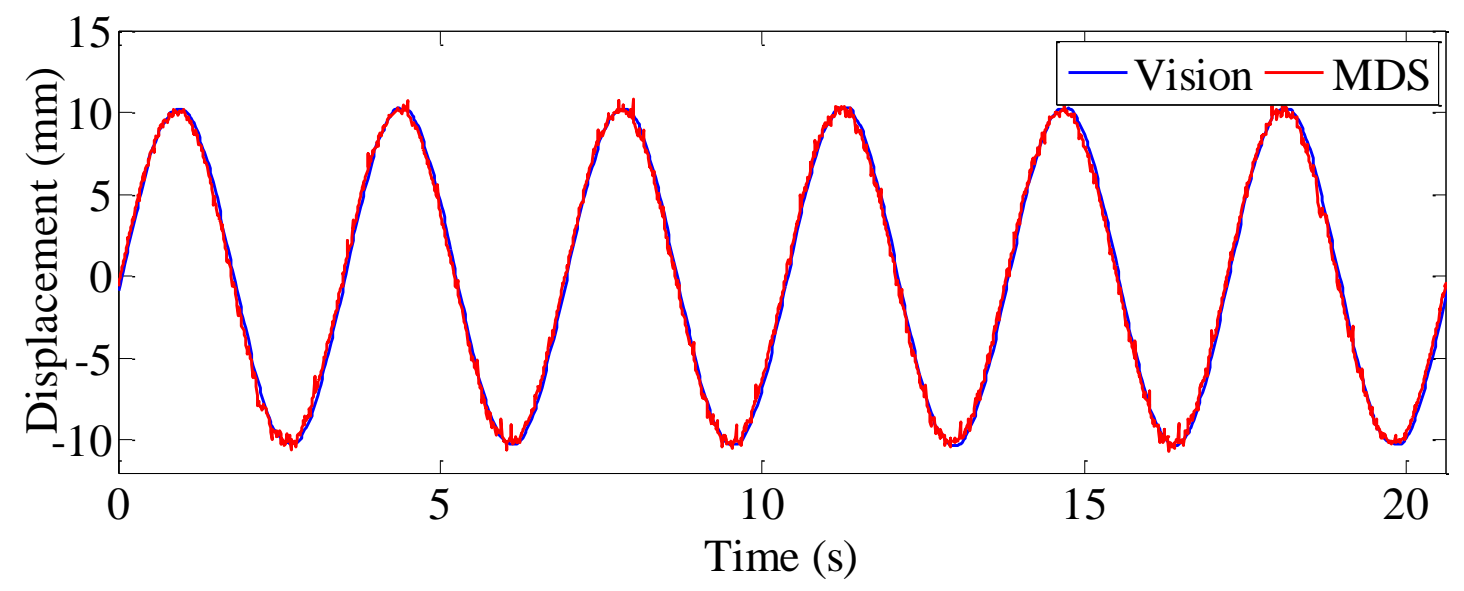

(a)

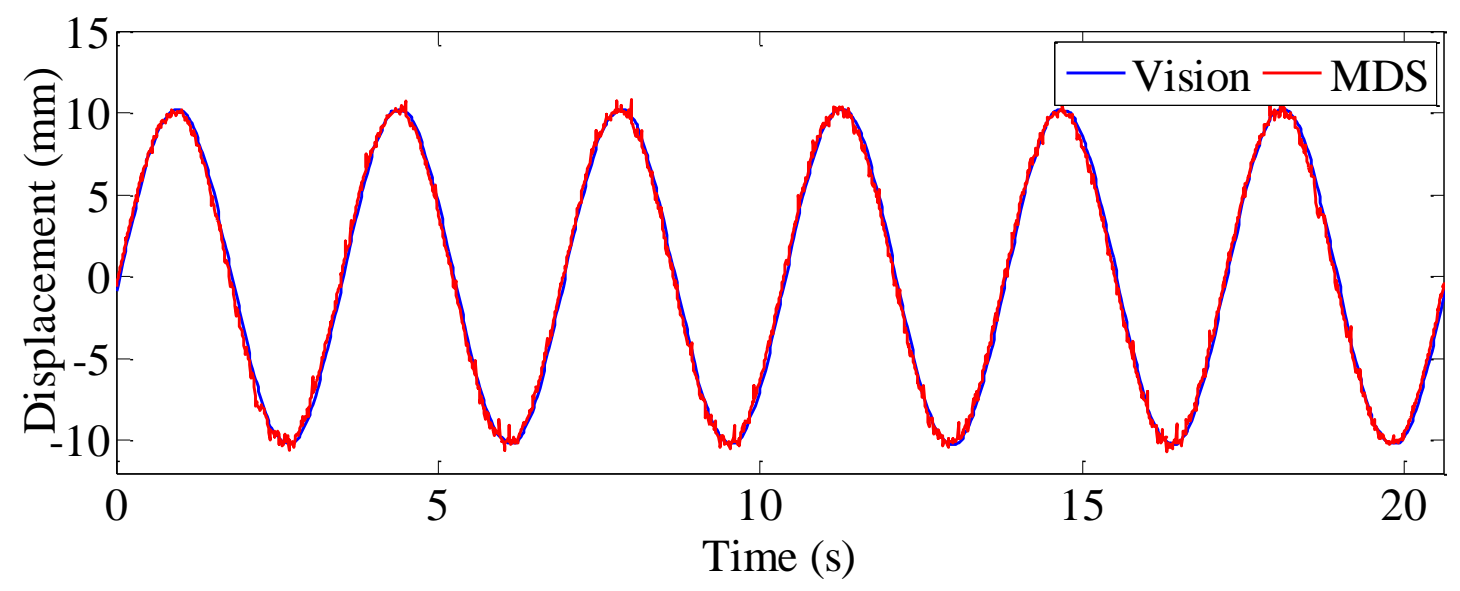

(b)

Fig. 9. Displacement time histories in case 1.4: (a) P1, (b) P2 


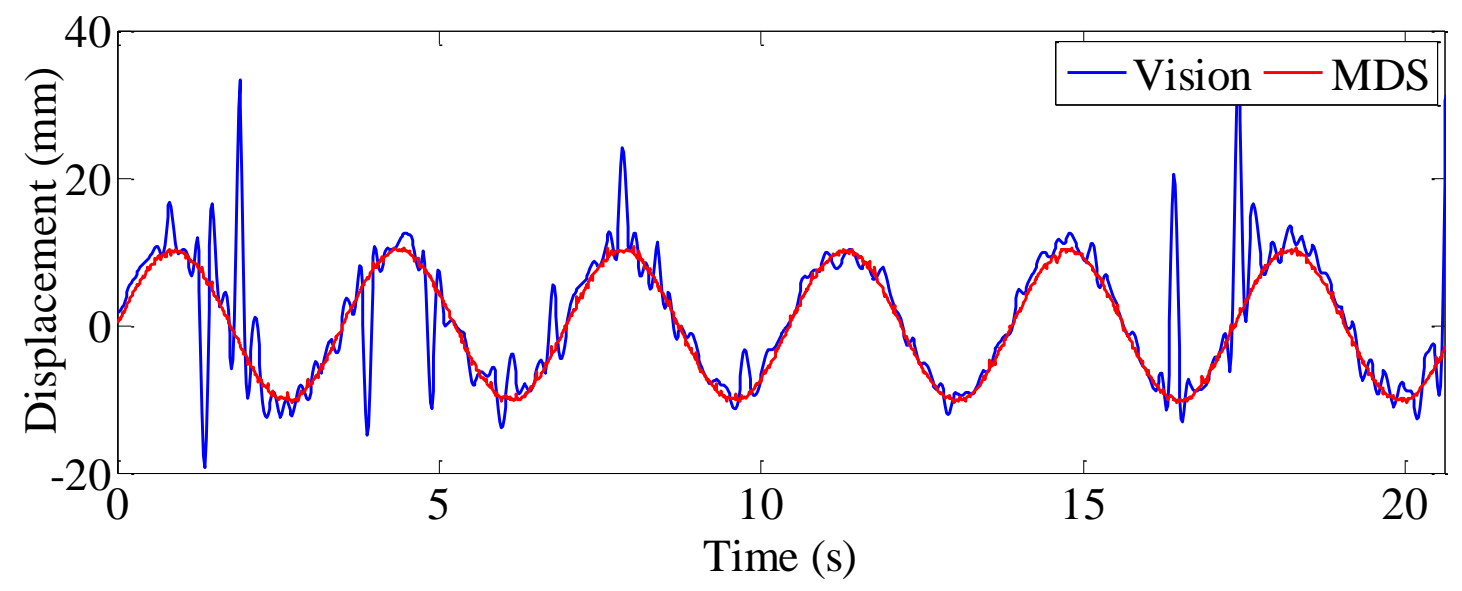

(a)

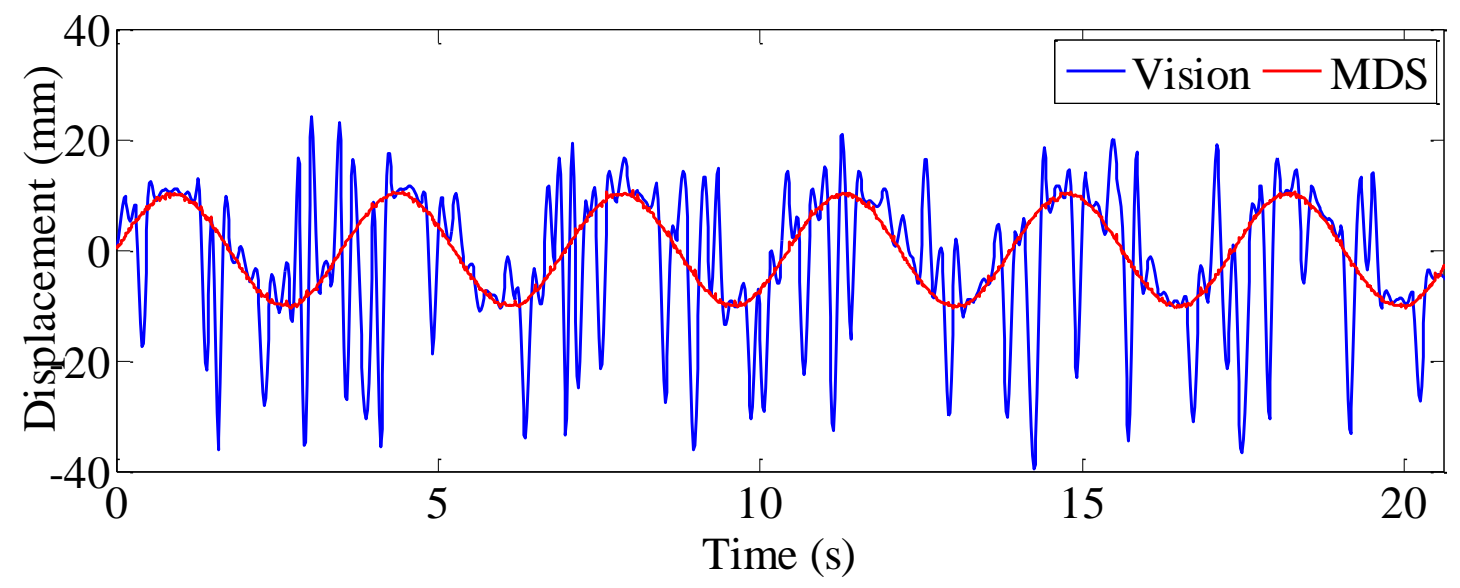

(b)

Fig. 10. Displacement time histories in case 1.5: (a) P1, (b) P2 

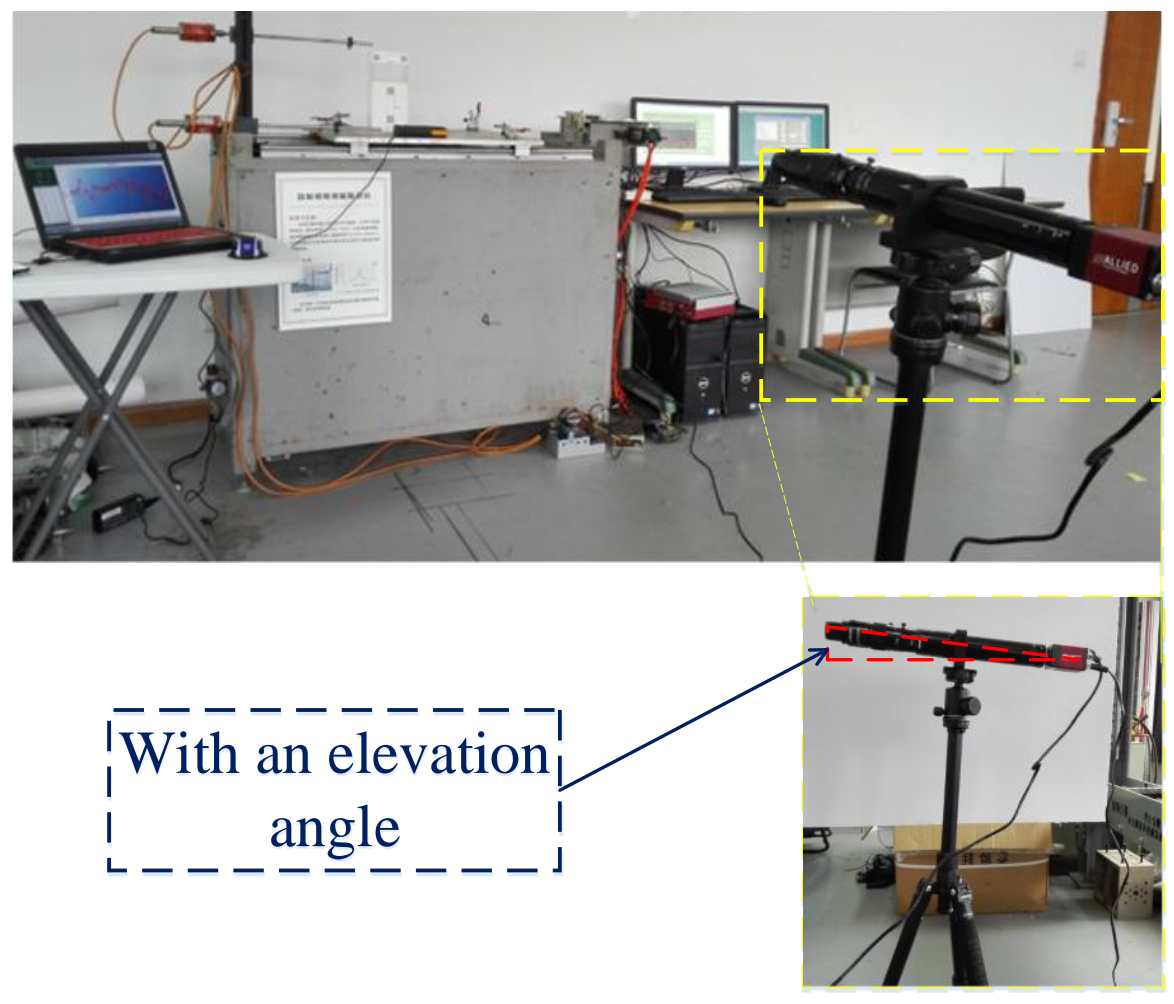

Fig. 11. Experimental setup for study of elevation angle effect 


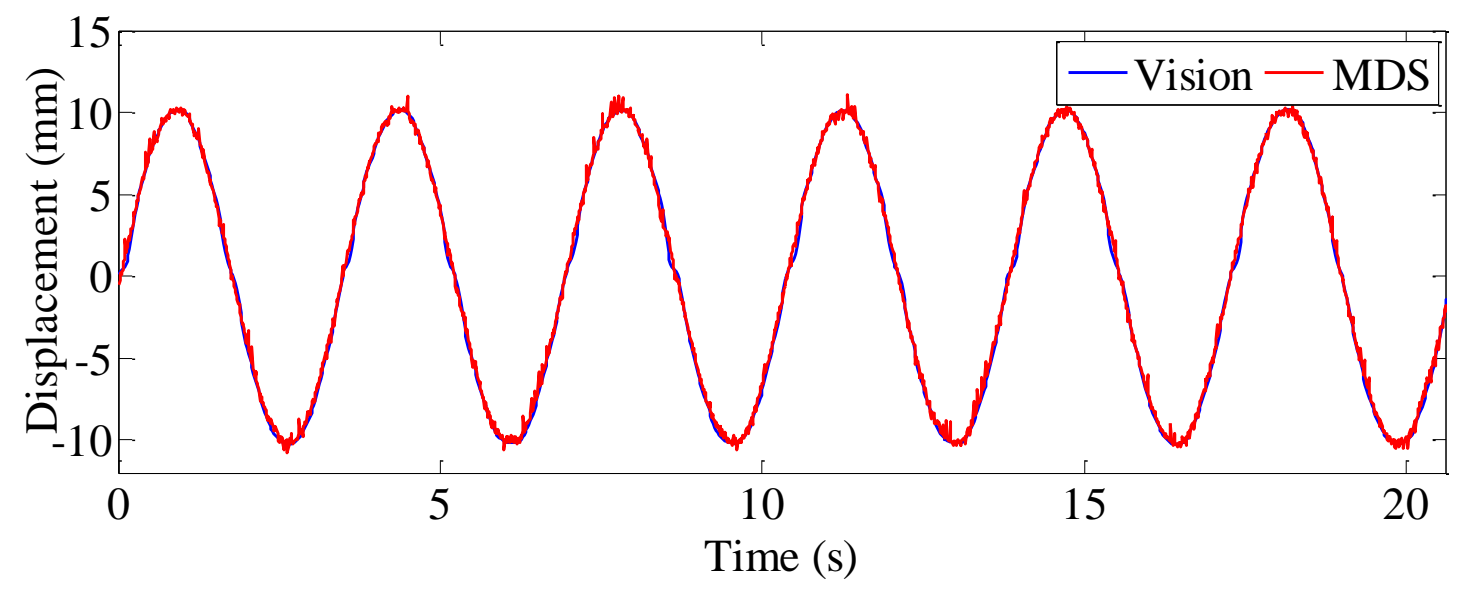

(a)

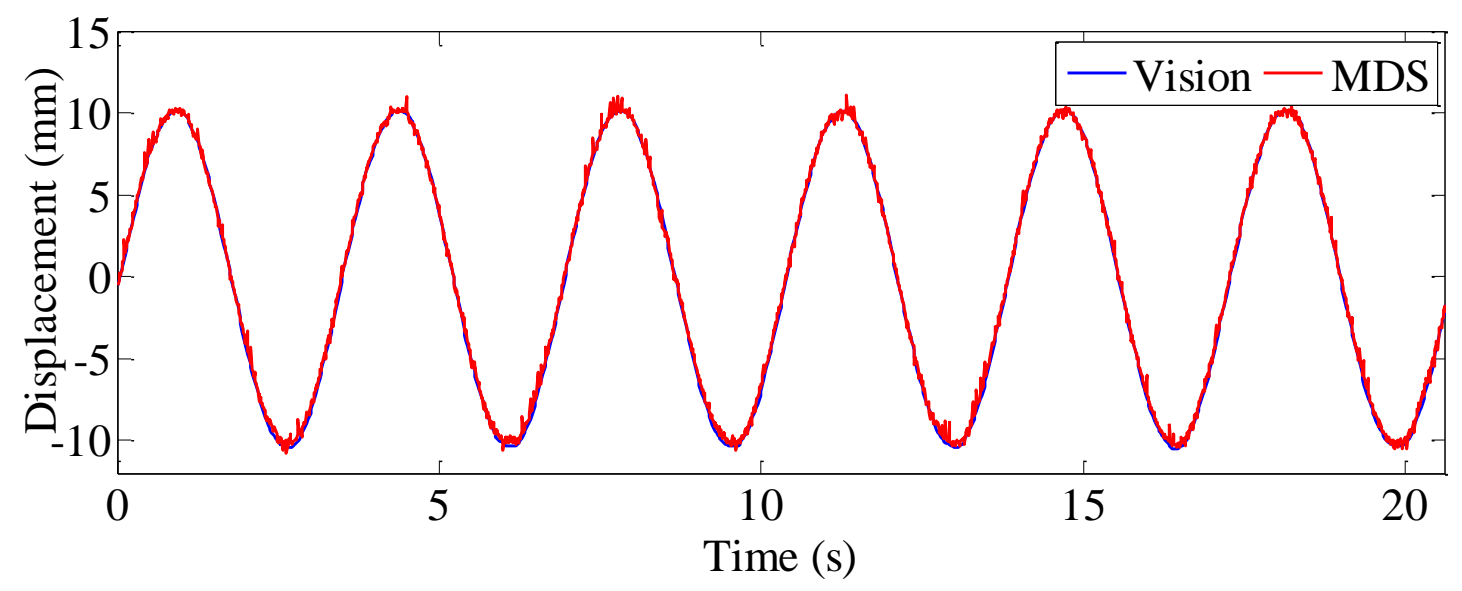

(b)

Fig. 12. Displacement time histories in case 2.1: (a) P1, (b) P2 


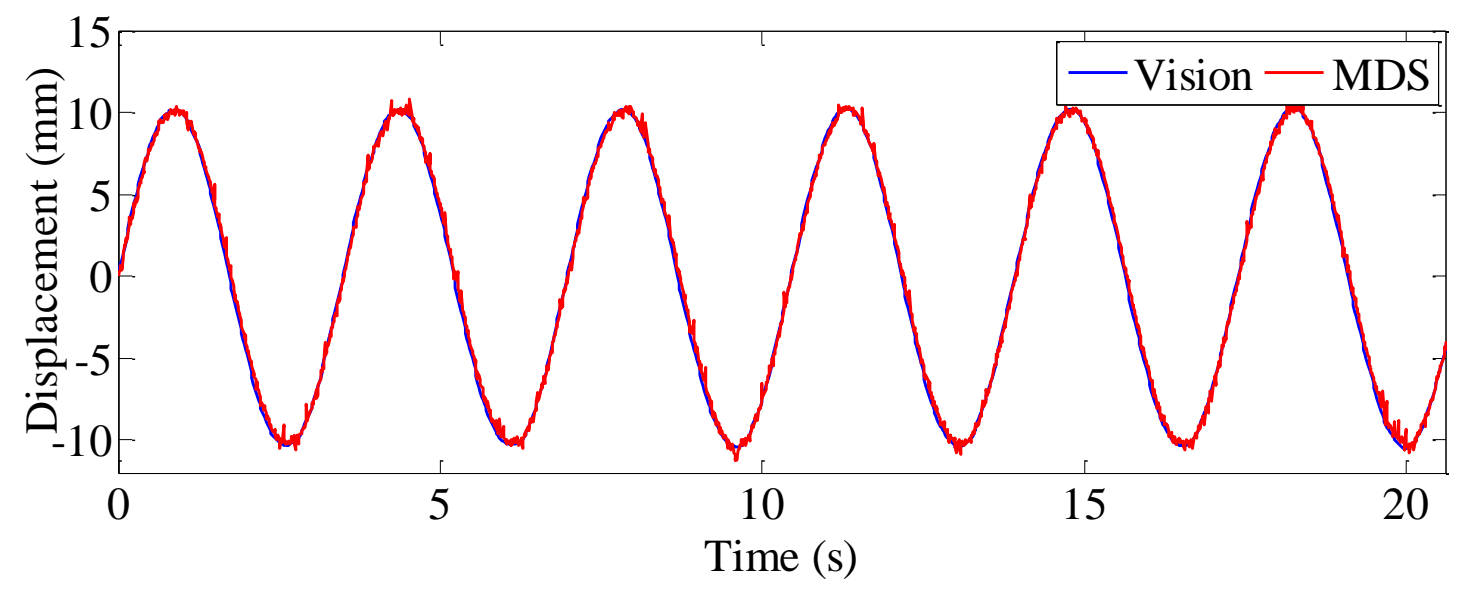

(a)

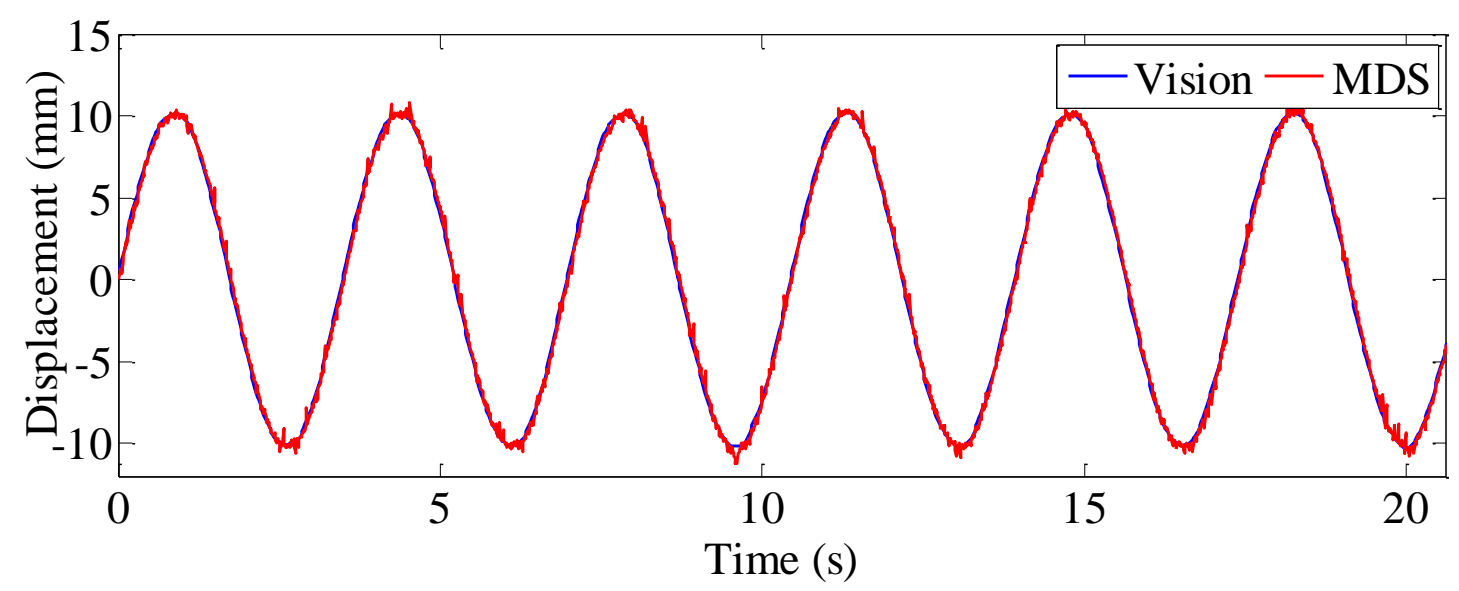

(b)

Fig. 13. Displacement time histories in case 2.2: (a) P1, (b) $\mathrm{P} 2$ 


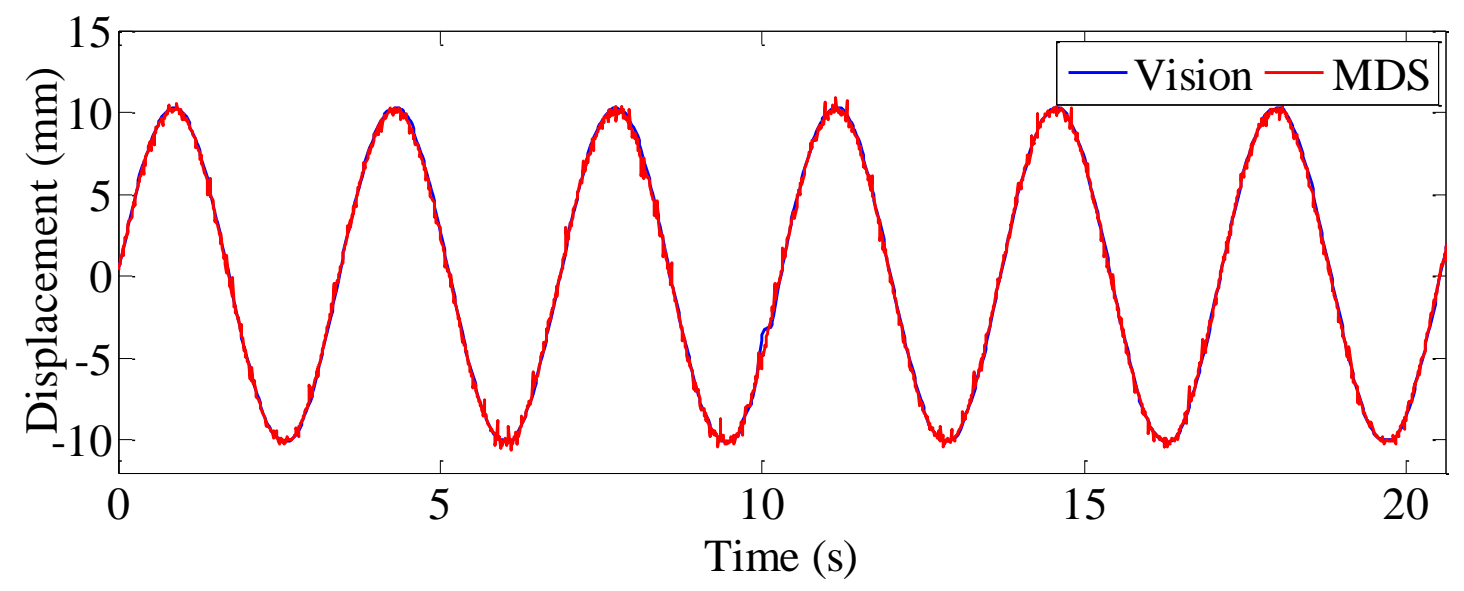

(a)

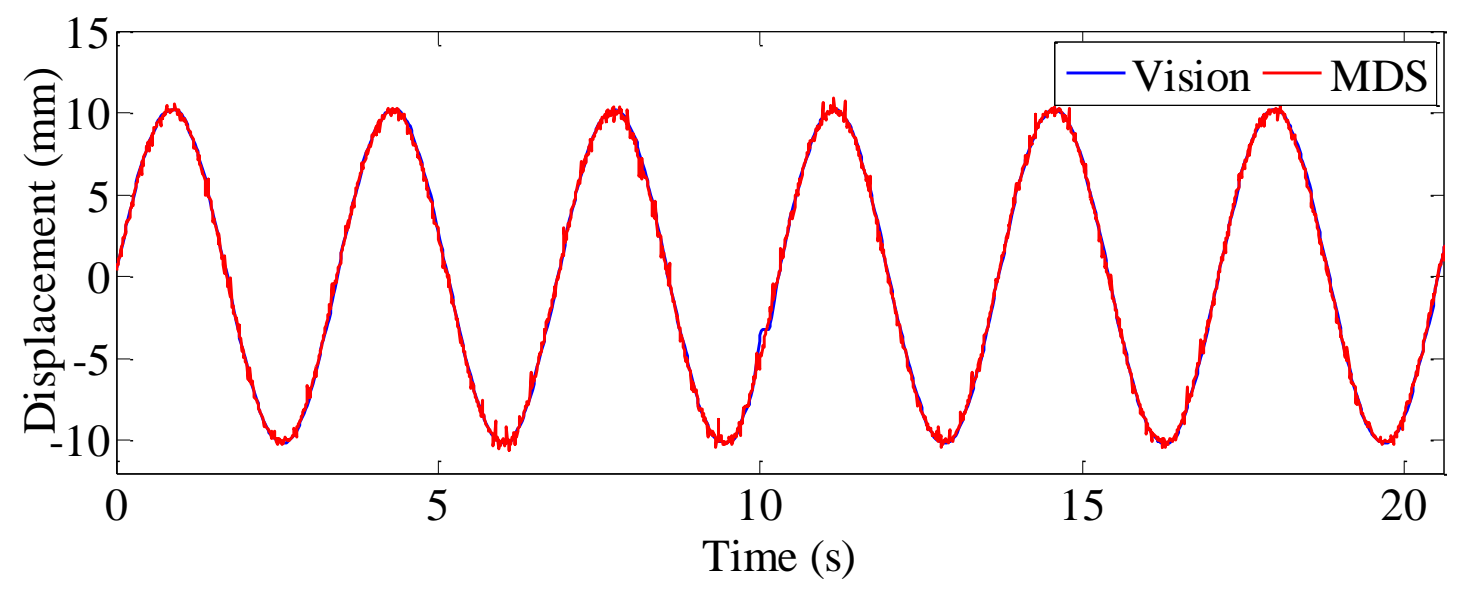

(b)

Fig. 14. Displacement time histories in case 2.3: (a) P1, (b) P2 


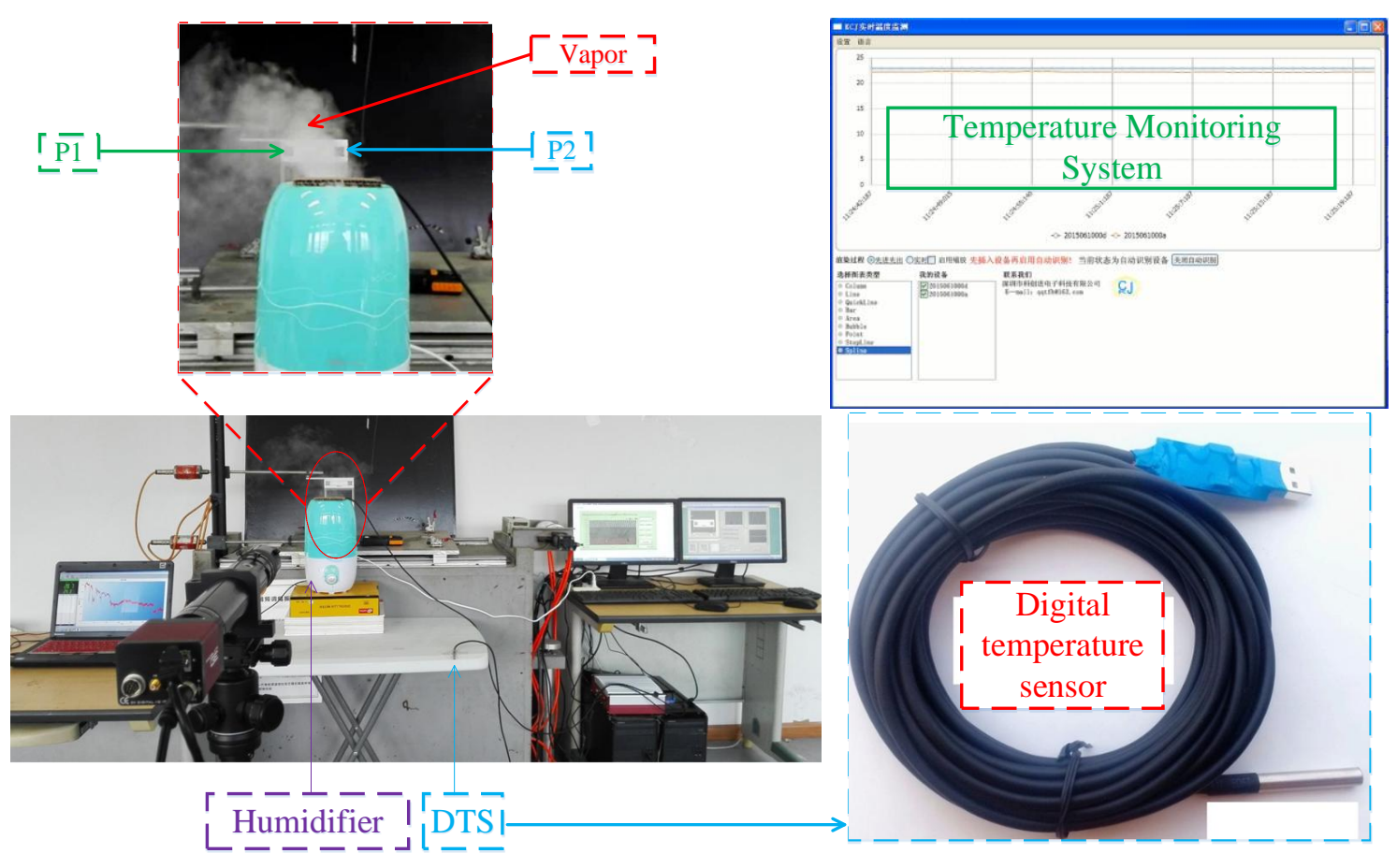

Fig. 15. Experimental setup for study of vapor effect 


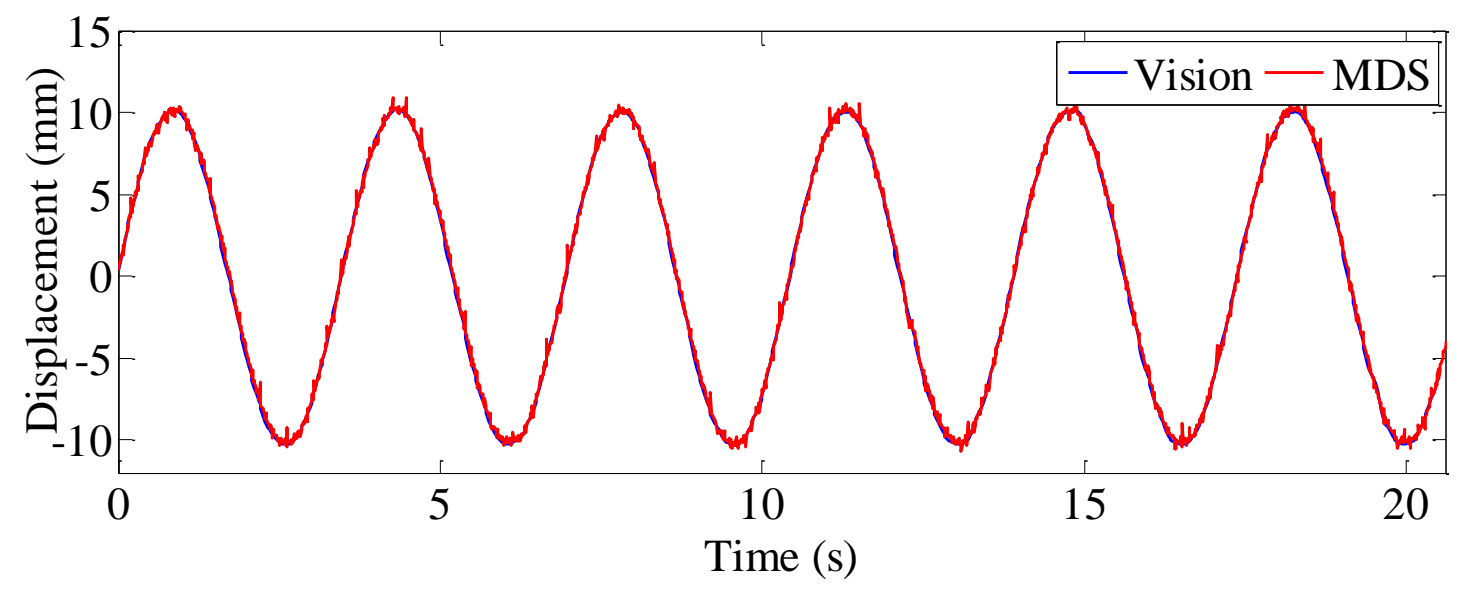

(a)

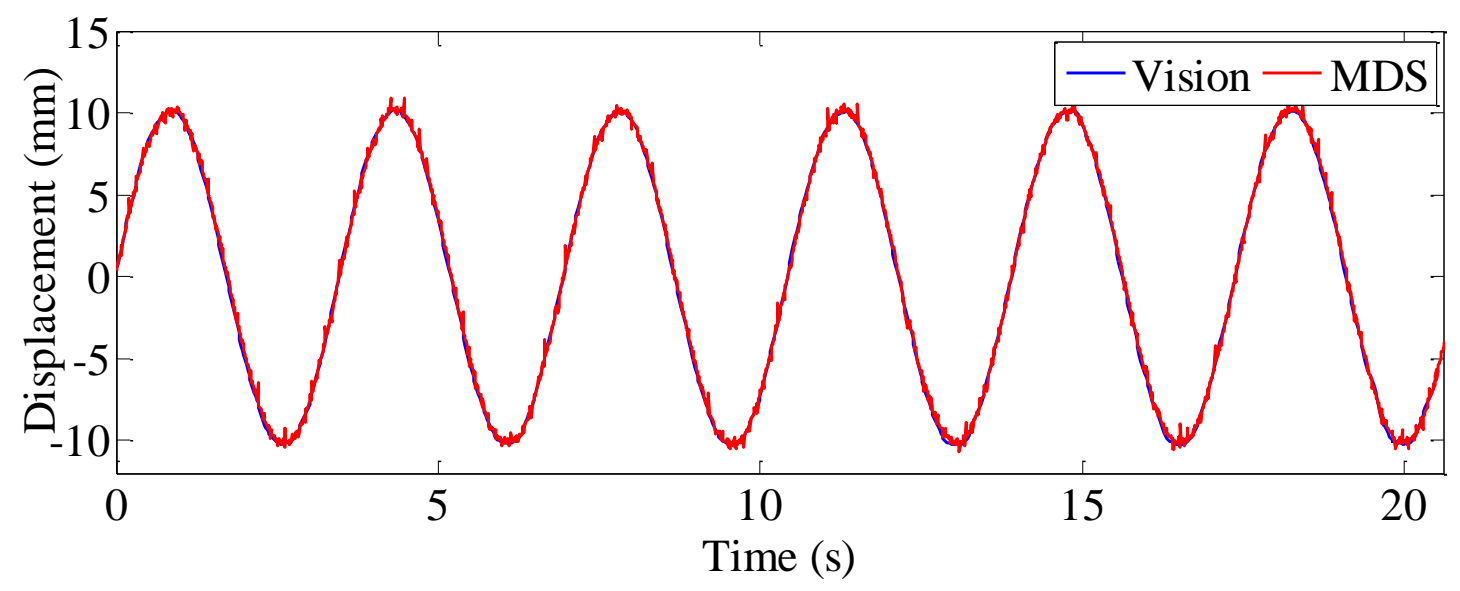

(b)

Fig. 16. Displacement time histories in case 3.1: (a) P1, (b) P2 


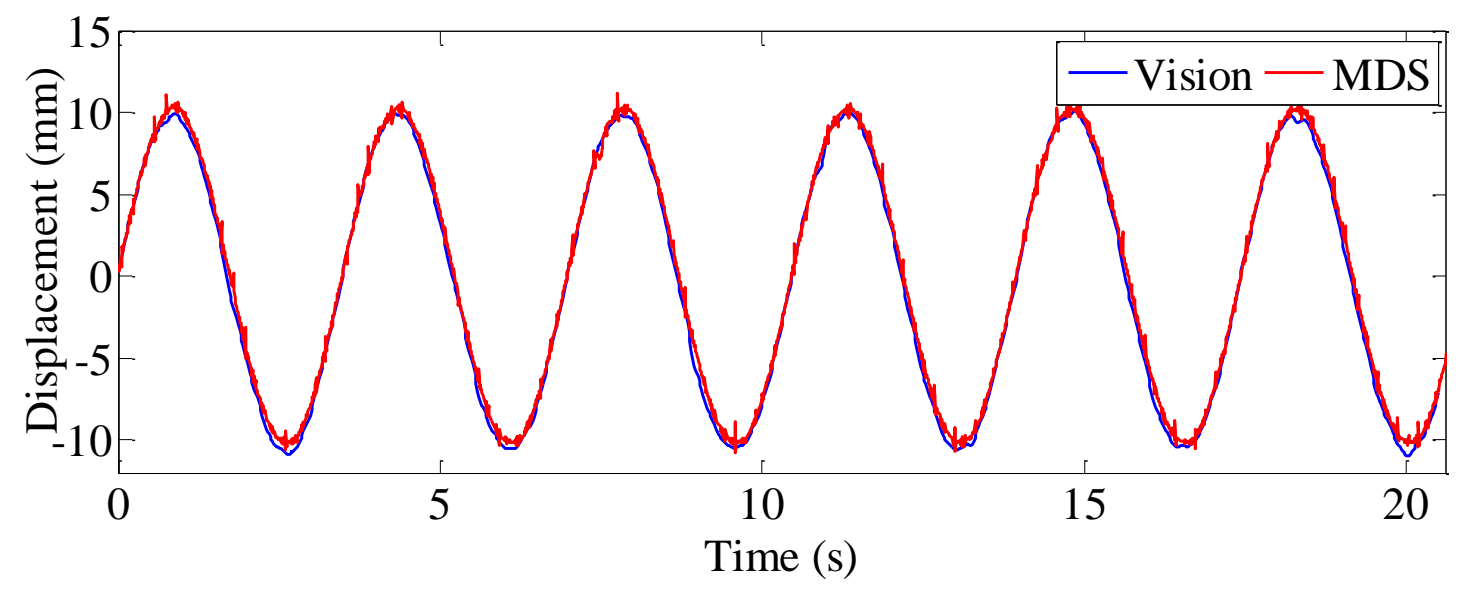

(a)

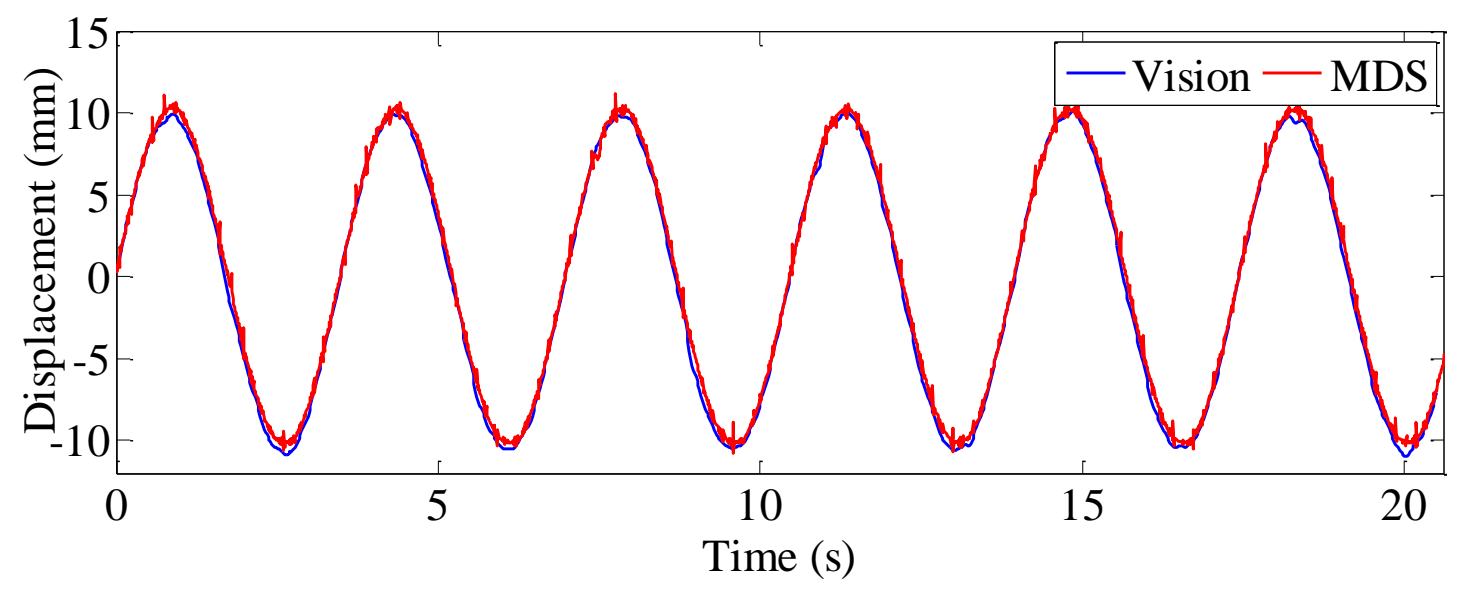

(b)

Fig. 17. Displacement time histories in case 3.2: (a) P1, (b) P2 


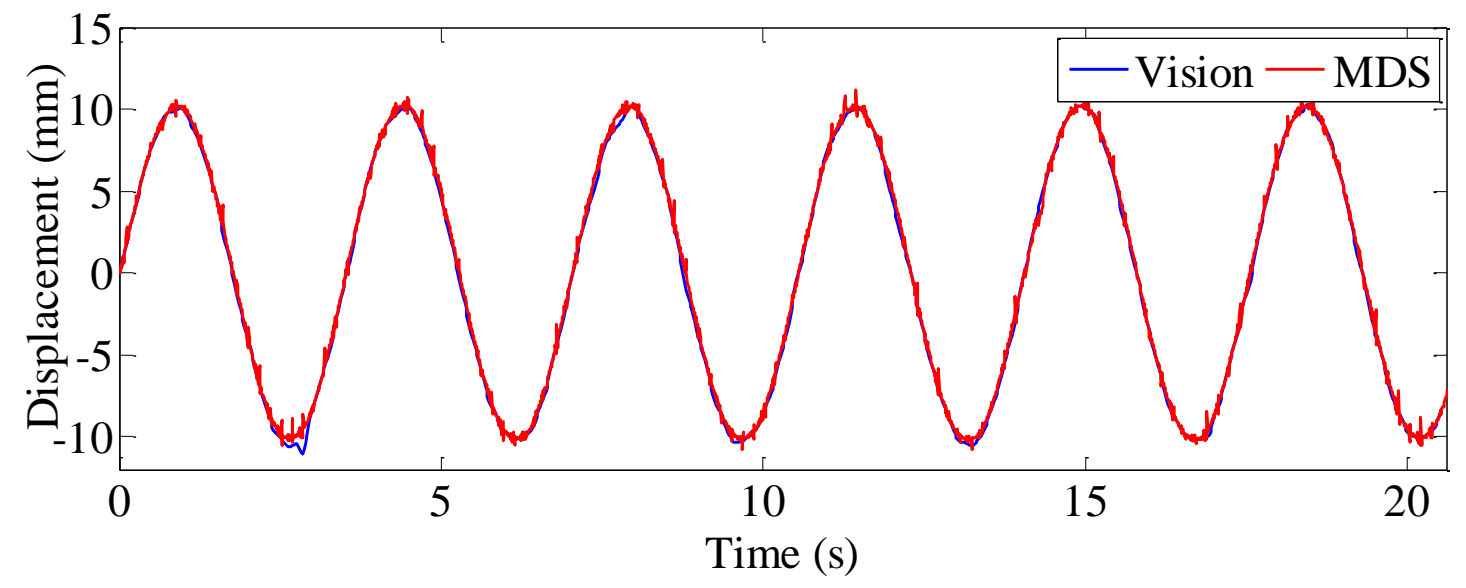

(a)

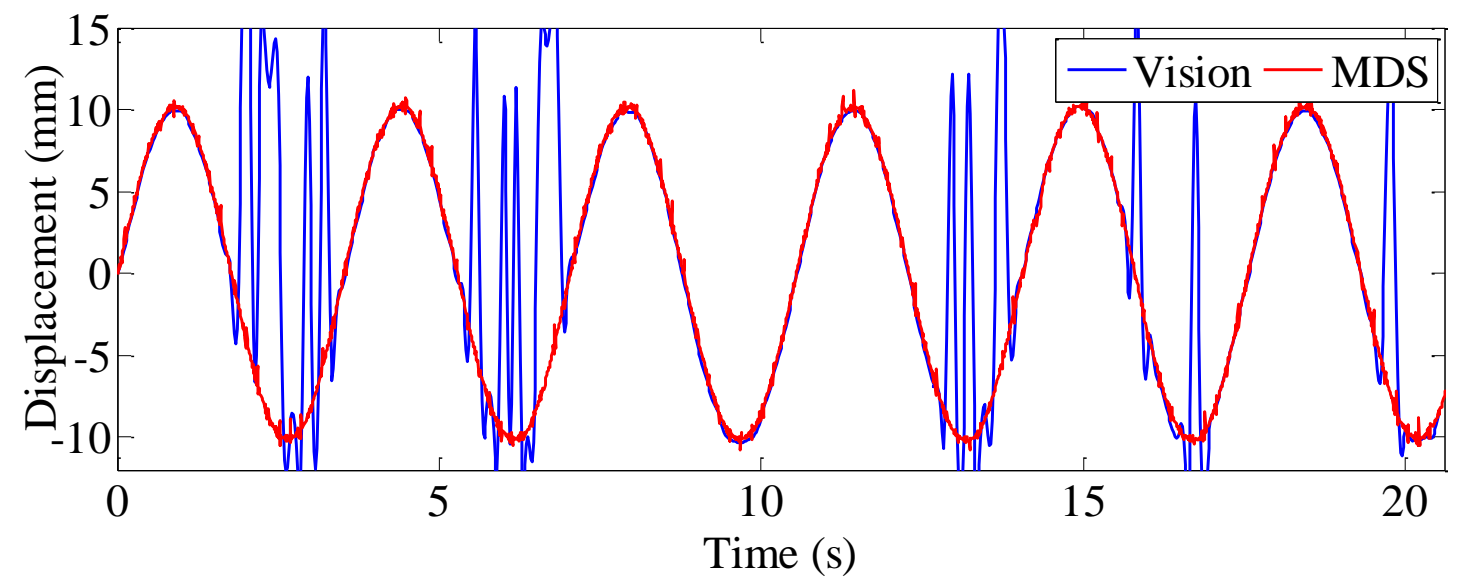

(b)

Fig. 18. Displacement time histories in case 3.3: (a) P1, (b) P2 


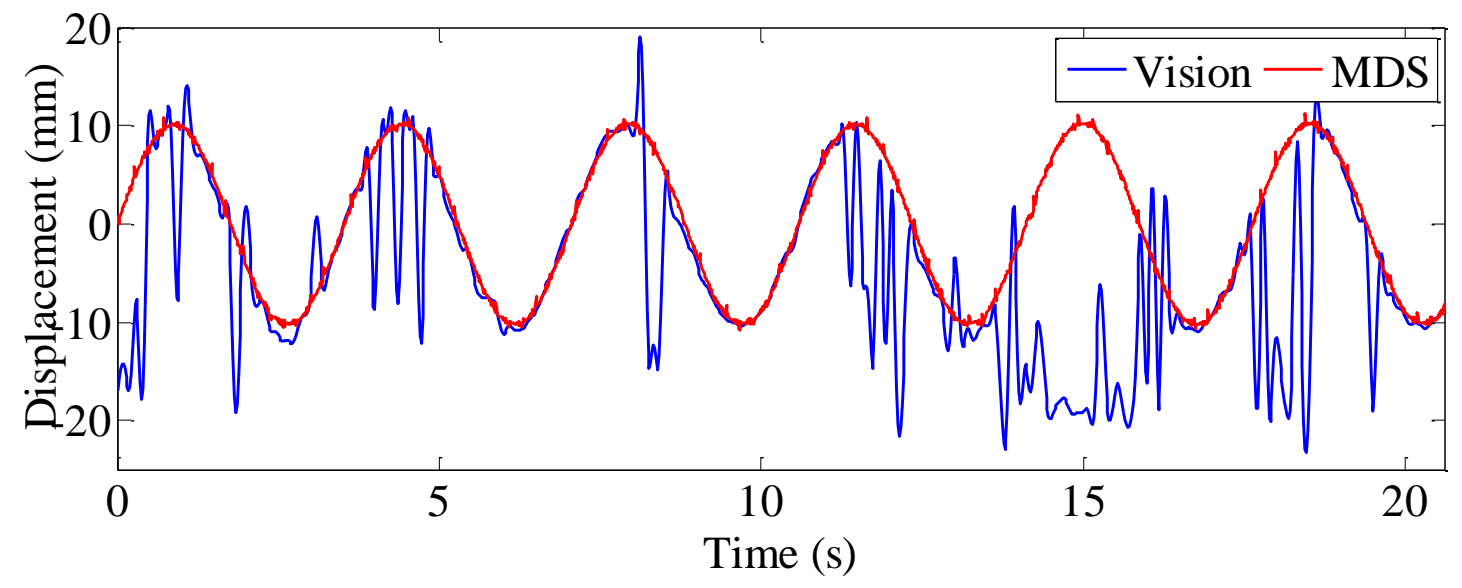

(a)

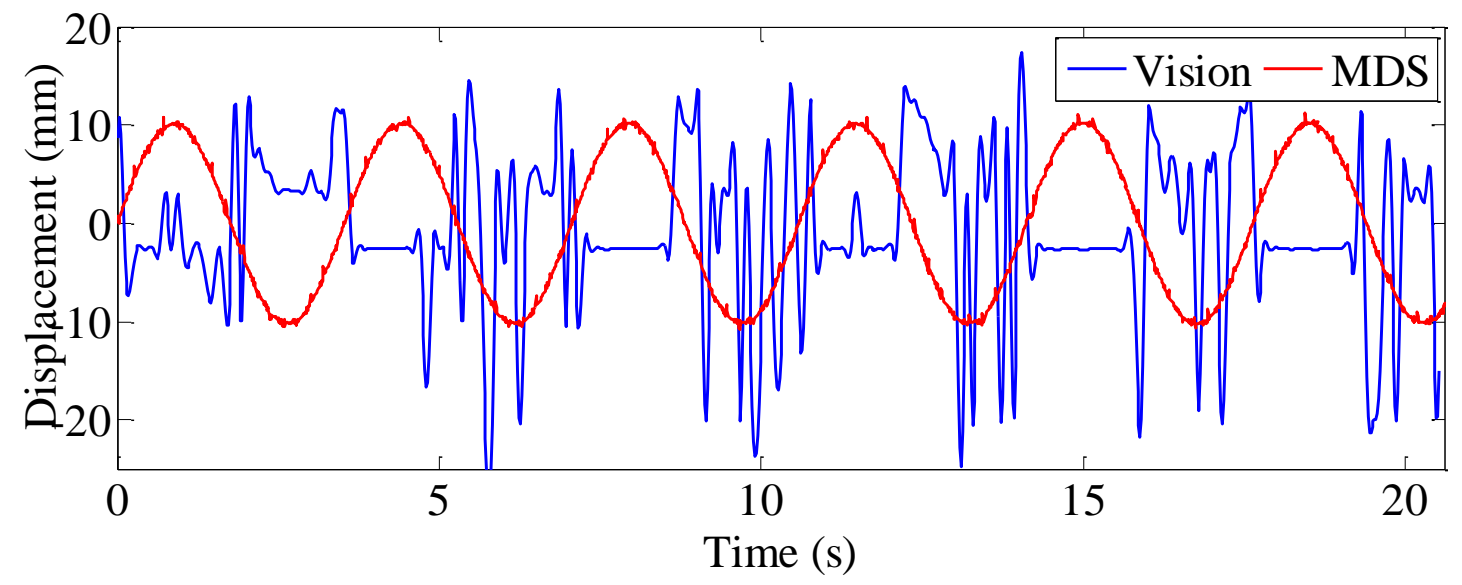

(b)

Fig. 19. Displacement time histories in case 3.4: (a) P1, (b) P2 


\section{List of Table Captions}

Table 1. Test cases for study of illumination effect

Table 2. Test cases for study of elevation angle effect

Table 3. Test cases for study of vapor effect 
Table 1. Test cases for study of illumination effect

\begin{tabular}{cc}
\hline Case & Level of illumination (lux) \\
\hline 1.1 & 600 \\
1.2 & 450 \\
1.3 & 300 \\
1.4 & 150 \\
1.5 & 75 \\
\hline
\end{tabular}


Table 2. Test cases for study of elevation angle effect

\begin{tabular}{cc}
\hline Case & Elevation angle of digital camera and lens $\left(^{\circ}\right)$ \\
\hline 2.1 & 5 \\
2.2 & 10 \\
2.3 & 15 \\
\hline
\end{tabular}


Table 3. Test cases for study of vapor effect

\begin{tabular}{ccc}
\hline Case & Level of vapor & Temperature difference $\left({ }^{\circ} \mathrm{C}\right)$ \\
\hline 3.1 & 1 & 0.19 \\
3.2 & 2 & 0.44 \\
3.3 & 3 & 1.62 \\
3.4 & 4 & 5.01 \\
\hline
\end{tabular}

